A N N A L E S Annales de Bretagne et des Pays de l'Ouest

\title{
L'Académie protestante de Saumur et les Néerlandais
}

The Protestant Academy of Saumur and the Dutch

\section{Willem Frijhoff}

\section{(2) OpenEdition}

1 Journals

Édition électronique

URL : http://journals.openedition.org/abpo/3429

DOI : $10.4000 /$ abpo.3429

ISBN : 978-2-7535-5365-1

ISSN : $2108-6443$

\section{Éditeur}

Presses universitaires de Rennes

Édition imprimée

Date de publication : 30 décembre 2016

Pagination : 61-91

ISBN : 978-2-7535-5363-7

ISSN : 0399-0826

\section{Référence électronique}

Willem Frijhoff, "L'Académie protestante de Saumur et les Néerlandais », Annales de Bretagne et des

Pays de l'Ouest [En ligne], 123-4 | 2016, mis en ligne le 30 décembre 2018, consulté le 03 janvier 2020. URL : http://journals.openedition.org/abpo/3429 ; DOI : 10.4000/abpo.3429 


\title{
L'Académie protestante de Saumur et les Néerlandais
}

\author{
Willem FRIJHOFF \\ Professeur émérite d'histoire moderne de l'université Libre d'Amsterdam - \\ Professeur invité à l'université Érasme de Rotterdam
}

L'attrait combiné du vin d'Anjou, de la pureté de la langue française, de la vigueur, puis de la modération théologique ont fait de Saumur une étape incontournable du grand et du petit tour des jeunes Néerlandais pérégrinant au XVII ${ }^{\mathrm{e}}$ siècle ${ }^{1}$. Contrairement à Angers, Bourges et surtout Orléans, où les étudiants venaient soit pour obtenir un grade facile soit, surtout s'ils étaient nobles ou avaient des prétentions aristocratiques, pour parader et se faire valoir dans les Nations Germaniques et autres structures de sociabilité réglée, Saumur était une petite ville où la convivialité était la règle et le protestantisme dominant, ce qui rendait la ville extrêmement populaire parmi les jeunes bourgeois néerlandais désireux d'apprendre l'esprit de la France sans se fatiguer outre mesure, tout en restant dans le droit fil religieux. L'attrait de Saumur était double : la ville se trouvait au cœur de la région réputée la plus agréable du royaume et la plus propice à l'apprentissage d'un français mélodieux, acceptable à la cour; et contrairement à quasiment toutes les autres villes populaires parmi les jeunes, elle était dotée d'une institution publique d'enseignement supérieur, l'académie protestante, qui était entourée à son tour de maîtres particuliers en toutes sortes de pratiques sociales, de la danse et la musique à l'équitation et au dessin. Pour les jeunes étrangers, l'académie n'était donc qu'un atout de Saumur parmi d'autres, et les malheurs que l'académie connut au cours de son existence n'eurent pas forcément une incidence directe sur la fréquentation de la ville ${ }^{2}$.

1. DiBon, Paul, « Le voyage en France des étudiants néerlandais au XvII ${ }^{\mathrm{e}}$ siècle ", in DiBon, Paul, Regards sur la Hollande du Siècle d'Or, Naples, Vivarium, 1990, p. 109-151.

2. BoDIN, Jean-François, Saumur : recherches historiques sur la ville, ses monuments et ceux de son arrondissement [fac-similé de l'édition de 1814], t. II, Paris, Le Livre d'histoire, 2003, p. 155-166, 217-232, 293-298; BouRchEnIN, Pierre Daniel, Étude sur les académies protestantes en France aux XVTe et XVIT siècles, Paris, Grassart, 1882, p. 137-146, 404-428; MÉTEYER, L.-Jules, L'Académie protestante de Saumur, Paris, Éd. La Cause, s.d. [1934]; 
Saumur se présentait comme une ville multifonctionnelle. Elle attirait par conséquent plusieurs catégories de jeunes : futurs pasteurs, étudiants, aristocrates au grand tour, touristes authentiques, simples passants. Quelques grands noms de l'histoire hollandaise ont laissé des témoignages de leur passage à Saumur et tout d'abord Pieter de la Court, qui y séjourna en mai et juin 1642. Ce jeune étudiant, fils d'un immigré wallon fabriquant de drap de Leyde, était un intellectuel observateur et brillant; il songea alors à se faire ministre mais finit sa vie comme un auteur politique influent d'esprit républicain, tenant du libéralisme économique. Au cours de son séjour il se montra surtout intéressé par la religion, réformée autant que catholique. L'autre exemple est Joan Huydecoper, fils d'un puissant bourgmestre d'Amsterdam et lui-même futur bourgmestre. Doué lui aussi, mais plutôt pour la vie politique et sociale, il constitue le prototype du touriste fortuné pour qui la religion n'était que la toile de fond ordinaire d'une réalité quotidienne autrement plus attrayante : les excursions en Anjou et dans le Saumurois, les chevaux et le jeu. D'août 1648 à avril 1649, il resta pendant neuf mois plus ou moins bloqué à Saumur par les turbulences de la Fronde et par le refus obstiné de son père de le laisser partir pour l'Italie - refus qu'il vainquit provisoirement par les efforts coordonnés des différents membres de sa famille et sa propre sagacité financière; mais, dès son arrivée à Genève, son père s'y opposa de nouveau, cette fois pour de bon. Le journal et les notes de voyage de de La Court et les lettres et relevés des dépenses de Huydecoper fournissent donc un excellent tableau des deux formes majeures d'interaction entre les jeunes étudiants ou voyageurs et la société saumuroise, et de la place de l'académie dans leur voyage.

\section{Académies et universités}

La nouvelle histoire internationale des universités, moins portée sur des critères institutionnels que par le passé, a réhabilité les académies protestantes qui, pour des raisons diverses, n'avaient pas réussi à se faire reconnaître comme universités à part entière et n'étaient donc pas habilitées à délivrer les doctorats nécessaires aux carrières juridiques et médicales et, pour certains postes, dans les Églises. Elle a souligné le potentiel universitaire que les meilleures d'entre elles ont su faire valoir et montré que leur enseignement et le climat des études ne le cédaient en rien à ceux des universités en titre ${ }^{3}$. Cela vaut certainement pour les

LEBRUn, François, "Entre Genève et Rome, Saumur au XVII siècle ", in MATIGnON, Bernard et LeBrun, François (dir.), L'Anjou, Paris, Autrement, 1988; LEBrun, François (dir.), Saumur, capitale européenne du protestantisme au XvII siècle [3 $3^{\mathrm{e}}$ Cahier de Fontevraud], Abbaye Royale de Fontevraud, 1991 ; LANDAIS, Hubert, Histoire de Saumur Toulouse, Privat, 1997, en particulier Poton, Didier, " Réforme et guerres de religion (1500-1621) ", p. 137-163, et Poton, Didier et LEBRUN, François, " Les temps de controverse (1621-1685) ", p. 165-185; [http://archives.ville-saumur.fr/am_saumur/app/03_archives_en_ligne/01_academie_protestante/presentation_academie.pdf].

3. De Ridder-Symoens, Hilde (dir.), A History of the University in Europe. Volume II : Universities in early modern Europe (1500-1800), Cambridge, Cambridge University 
académies de Die, Montauban, Saumur, Sedan et Strasbourg en France, pour celles de Herborn, Brême, Steinfurt, Hamm, Marburg, Heidelberg et Hanau en Allemagne, de Lausanne et Genève en Suisse, ou de Deventer et Amsterdam dans la République des Provinces-Unies. Les contemporains ne s'y sont d'ailleurs pas trompés. Les jeunes Néerlandais allaient étudier tour à tour dans les universités et les académies (appelées ici et là gymnases académiques, et dans les Provinces-Unies écoles illustres), quitte à clore leur périple par un passage ultra-rapide dans une vraie université, une " université fameuse " comme on disait alors, telles Orléans, Angers, Caen, Bourges, Valence, Padoue, Sienne, Duisburg ou Harderwijk, pour décrocher leur doctorat dans un temps record et moyennant finance, souvent dès le lendemain de leur arrivée. Les académies protestantes se targuaient d'ailleurs de posséder le droit de délivrer des maîtrises ès arts, même si les universités (catholiques) en titre le leur disputaient; à Saumur, ce diplôme pouvait être dispensé à la fin du curriculum du collège de plein exercice qui constituait l'infrastructure éducative du binôme collège-académie.

Une remarque similaire vaut pour les professeurs. Dans mainte carrière enseignante l'emploi au sein d'une académie s'insère harmonieusement dans une carrière par ailleurs toute universitaire. Il en est ainsi de celle des deux Hollandais qui ont enseigné à Saumur, le théologien Franciscus Gomarus, Gomar ou Gomard (1563-1641) et le philosophe Franco Petri Burgersdijck (1590-1635). Gomarus, le défenseur du calvinisme prédestinatianiste sévère, avait en 1611 quitté son poste de professeur de théologie à Leyde pour rejoindre le nouveau collège théologique de Middelbourg en Zélande, qui périclita rapidement. En octobre 1614, il accepta l'offre insistante d'un poste de professeur à Saumur pour le salaire extraordinaire de 1200 livres; arrivé le 17 mai 1615, il fut deux fois recteur, mais des problèmes avec ses lettres de naturalité, un conflit avec le " pape des huguenots " Philippe Du Plessis-Mornay (1549-1623), et ses propres ambitions comme patriarche de l'orthodoxie dans les Provinces-Unies protestantes le persuadèrent d'accepter une nomination à la nouvelle université de Groningue, qui avait fait appel à lui par l'intermédiaire de Festus Hommius, le régent orthodoxe du Collège théologique des États à Leyde. Il rentra dans la République en avril 1618 ${ }^{4}$. Burgersdijck de son côté entreprit, après ses études à Leyde, un grand tour qui le conduisit d'abord à Saumur et, au lieu de poursuivre son voyage vers l'Allemagne comme cela avait été son intention, il y demeura cinq ans. Pendant la demi-année de ses études en théo-

Press, 1996. Sur les universités des Provinces-Unies, voir dernièrement FRJJHOFF, Willem, "Autonomie, monopole, concurrence : le facteur urbain dans la construction du réseau universitaire dans les Provinces-Unies ", in Amalou, Thierry, NoguĖs, Boris (dir.), Les Universités dans la ville, XVI-XVII siècle, Rennes, PUR, 2013, p. 25-49.

4. Archives municipales de Saumur, Papier et regestre des affaires de l'académie royale establie à Saumur depuis le mois d'octobre 1613 (-1685) [transcription dans http://archives. ville-saumur.fr/am_saumur/app/03_archives_en_ligne/01_academie_protestante/viewer. php], f $\mathrm{f}^{\circ} 11 \mathrm{r}^{\mathrm{o}}-30 \mathrm{r}^{\mathrm{o}}$, passim; notice biographique dans Nieuw Nederlandsch Biografisch Woordenboek, t. VII, Leyde : Sijthoff, 1927 / reprint Amsterdam, N. Israel, 1974, col. 483-486. 
logie à la nouvelle académie, notamment auprès de logicien Marc Duncan (vers 1570-1640), il se fit remarquer par Du Plessis-Mornay qui, le 9 juillet 1615 , le fit nommer professeur de philosophie, en dépit du fait qu'il n'avait encore obtenu aucun grade universitaire. Fin 1619, il échangea cette fonction pour un poste de professeur de logique à l'université de Leyde, qui se hâta de lui conférer le titre de magister artium et où il fit école par ses ouvrages d'un aristotélisme modernisé ${ }^{5}$.

Dans la même année 1615, le jeune Merula se porta candidat à la régence de la II $^{\mathrm{e}}$ puis de la III $^{\mathrm{e}}$ classe du collège protestant de Saumur. Il s'agit sans doute de Willem Merula, âgé d'une vingtaine d'années, qui avait été étudiant à Leyde en 1612 et qui avait publié en 1614 la chronique historique (Tijdt-Thresoor) de feu son père Paullus Merula (1558-1607), juriste et historiographe de la Gueldre ${ }^{6}$. En raison de la timidité dont il fit preuve lors de son examen d'admission et de son manque supposé d'autorité, et tout en recevant un témoignage vibrant de ses capacités, il ne fut pas admis. Mais il n'y avait pas que des étudiants à Saumur. Quatre ans après l'échec de Merula, le 18 février 1619, des poursuites furent engagées contre le proposant Jean Bachellé de Metz et François van Lieshout, natif d'Amsterdam, compagnon relieur dans la boutique de Thomas Portau, imprimeur de l'académie ${ }^{7}$ : ils avaient publié un libelle diffamatoire intitulé Satire démocritique contre les Écossais à l'académie. Le libelle fut détruit, et Van Lieshout dut publiquement demander pardon avant d'être licencié par Portau. Cela ne l'empêcha pas d'ouvrir quelques années plus tard un atelier d'imprimerie sous l'enseigne "In 't Groot Boeck" ("Au Grand Livre ") sur la place principale d'Amsterdam, le Dam, qui fonctionna de 1625 jusqu'à son décès en $1646^{8}$.

Initialement, la ville de Saumur s'insérait donc harmonieusement dans le circuit d'études supérieures et les perspectives de carrière éducative des jeunes protestants néerlandais, sans doute le plus souvent en théologie, mais parfois aussi dans les humanités, en philosophie ou en mathéma-

5. Arch. mun. de Saumur, Papier et regestre des affaires de l'académie royale, $\mathrm{f}^{\mathrm{o}} 17 \mathrm{r}^{\mathrm{o}}$, $20 r^{\circ}, 39 r^{\circ}, 40 r^{\circ}, 49 r^{\circ}$. Notice biographique : Nieuw Nederlandsch Biografisch Woordenboek, t. VII, col. 229-231; DiBon, Paul, "Le voyage... ", art. cit., p. 117. Sur son aristotélisme : VAn Bunge, Wiep, " Philosophy ", in FrIJHOFf, Willem, SPIES, Marijke (dir.), 1650: Hard-Won Unity, [Dutch Culture in a European Perspective], Assen, Royal van Gorcum/Basingstoke: Palgrave Macmillan, 2004, p. 283-286, 306-307.

6. Arch. mun. de Saumur, Papier et regestre des affaires de l'académie royale, $\mathrm{f}^{\circ} 14 \mathrm{r}^{\mathrm{O}}$ $14 \mathrm{v}^{\mathrm{o}}$. Notice biographique : Nieuw Nederlandsch Biografisch Woordenboek, t. II (1912), col. 904 .

7. Arch. mun. de Saumur, Papier et regestre des affaires de l'académie royale, $\mathrm{f}^{\circ} 37 \mathrm{r}^{\mathrm{o}}$ $37 \mathrm{v}^{\mathrm{o}}$. Bourchenin, Pierre Daniel, Étude sur les académies protestantes..., op. cit., p. 386 , n'a pas compris ce passage et a mal identifié le nom du compagnon relieur. L'officine de Portau fut saccagée par les troupes en 1621, et il a dû quitter la ville. Un Thomas Portau, probablement son fils, fut admis en 1648 comme chirurgien ordinaire dans la ville de Zutphen en Gueldre, où il eut une descendance. En 1657 il fut nommé chirurgien-général de la marine de guerre de la République, mais mourut peu après. Son fils Thomas (1657vers 1708) étudia le droit à Leyde mais s'établit comme négociant en grains à Zutphen.

8. GRuYs, Jan Albert et Bos, Jan, Adresboek Nederlandse Drukkers en Boekverkopers tot 1700, La Haye, Koninklijke Bibliotheek, 1999, p. 83. 
tiques $^{9}$. Cela valait également pour certains catholiques de la République car l'académie protestante n'était pas la seule institution théologique à Saumur. Conçue dès 1592 par Du Plessis-Mornay, sa fondation en tant qu'université protestante avait été prévue en 1598 par le synode national de Montpellier et décidée par le synode de Jargeau en 1601 mais réalisée seulement en 1607 comme superstructure du collège protestant ouvert en 1599. On y enseignait d'ailleurs également la philosophie et les mathématiques, et plus épisodiquement le droit et la médecine. Mais la ville était restée majoritairement catholique et dans un esprit de paix de religion pragmatique, le climat de controverse religieuse y tournait tout entier autour du problème de la grâce, parmi les protestants comme - on l'oublie souvent - parmi les catholiques. Ainsi a-t-on pu appeler le couvent de la Fidélité qui se trouvait au centre-ville un "Port-Royal saumurois " : sous la prieure Madeleine Gautron, élue en 1634 à l'âge de 23 ans, ce couvent vécut pendant plus de quarante ans dans un régime rigoriste d'une extrême austérité, sanctionnée par ses confesseurs de la congrégation de l'Oratoire ${ }^{10}$. En 1649 une deuxième institution théologique fut érigée à Saumur auprès du vieux sanctuaire de Notre-Dame-des-Ardilliers situé hors la ville, sur le bord de la Loire, qui était géré par la même congrégation de l'Oratoire depuis son installation dans cette résidence en 1619. C'était en même temps l'école théologique (ou grand séminaire) de l'Oratoire, fondée pour fournir un contrepoids à l'académie réformée par François Bourgoing, supérieur général de la congrégation mais aussi ancien supérieur de la maison de Saumur. Devenue dès sa fondation un haut lieu du rigorisme catholique, puis du jansénisme naissant, et resté tel sous le très long épiscopat (16491692) de l'évêque d'Angers Henry Arnauld, frère aîné du grand Antoine Arnauld, ce séminaire fut transféré à Montmorency en 1720 après la censure d'une thèse janséniste de théologie estimée dangereuse ${ }^{11}$.

Dès avant la fondation de cette académie catholique, le collège royal de Saumur, fondé vers 1615 et confié à l'Oratoire depuis 1624, était couplé avec la maison des Ardilliers. Collège de plein exercice, il faisait fonction de lieu d'études et d'enseignement théologique dans un esprit de controverse ${ }^{12}$.

9. Le catalogue des élèves du collège manque, mais on reconnaît dans le registre de l'académie le nom d'un des nombreux " fils de feu Monsieur de Saumaise " qui remporta le 4 novembre 1657 le second prix de prose latine et le prix du thème en grec. Son père Claude de Saumaise (1588-1653) avait été depuis 1631 professeur et chercheur à Leyde (Arch. mun. de Saumur, Papier et regestre des affaires de l'académie royale, $\mathrm{f}^{\circ} 169 \mathrm{r}^{\circ}$ ). Reinerus Neuhusius, fils d'Edo, le très savant recteur de l'école latine de Leeuwarden a été un " vrai " étudiant néerlandais d'humanités à Saumur. Voir la correspondance citée dans P. van Tuinen, " "A deo haec est vocatio" (Fan God komt dit beroep) ", It Beaken, t. 44 (1982), p. 97-116.

10. Delaunay, Louis, Un Port-Royal saumurois : les religieuses bénédictines de la Fidélité, Angers, J. Siraudeau, 1917.

11. Voir pour le contexte : MAILLARD, Jacques, L'Oratoire à Angers aux XVII et XVII siècles, Paris, Klincksieck, s.d. [1975].

12. Mise au point sur le collège catholique dans COMPÈRE, Marie-Madeleine et JuLIA, Dominique, Les Collèges français, $16^{e}-18^{e}$ siècles. Tome $2:$ Répertoire, France du Nord et de l'Ouest, Paris, INRP-CNRS, 1988, p. 594-599. 
Dès 1638, dans son répertoire des institutions religieuses, le chanoine anversois Aubert Le Mire (Miraeus) le confirme expressément pour la maison de l'Oratoire de Saumur qui hébergeait alors une trentaine de prêtres, "Theologiam, atque in primis Fidei controversias, adversus Calvinianos Salmurij potissimum obstrepentes, diligenter explicant ${ }^{13}$. " Il s'agit dans ce cas d'une première chaire de théologie, attestée dès 1633 dans cette " maison d'études ", comme les oratoriens l'appelaient. L'enseignement fut continué et établi sur un plan supérieur en 1649 dans l'école théologique des Ardilliers, lieu de rencontre des catholiques et protestants débattant sur les points de controverse, en particulier la justification et la grâce, sous l'impulsion du père Louis Thomassin arrivé en $1648^{14}$.

Mais dès les années 1640 un cursus d'enseignement formel, sous forme de séminaires, y est organisé pour les jeunes étudiants en philosophie et théologie de l'Oratoire. On y retrouve plusieurs jeunes Néerlandais catholiques de bonne famille entrés à l'Oratoire de Paris ou de Louvain : ainsi, dès 1636, Pierre Pelt d'Utrecht y étudie avec les professeurs Jean Berthad et Léonor de La Barde ${ }^{15}$. En 1645, il n'y a pas moins de quatre étudiants néerlandais dans cette école, auxquels s'ajoutent en 1646 un étudiant laïque et surtout le jeune oratorien Jean [Johannes] van Neercassel (1625-1686), futur évêque in partibus infidelium de Castorie et vicaire apostolique jansénisant de la Mission de Hollande, un vrai rassembleur et leader spirituel, mort en odeur de sainteté. Ayant fini ses études de théologie à Saumur avec Thomas Bély, il y enseigna lui-même pendant trois ans, de 1647 à 1650, la philosophie avec Jean Berthad puis la théologie avec Louis Thomassin. C'est à Saumur que ce futur prélat apprit les finesses de cette controverse qui par la nécessité d'un contact fréquent et d'un débat compréhensif avec l'adversaire était propice aux tendances jansénisantes. Dans la situation inverse de la République des Provinces-Unies, celles-ci continuaient de caractériser les positions de Neercassel et de son entourage clérical, voire même laïque ${ }^{16}$.

13. MiRAEUs, Aubertus, Regulae et constitutiones clericorum in congregatione viventium, Anvers, Trognesius, 1638, p. 126.

14. Bourchenin, Pierre Daniel, Étude sur les académies protestantes..., op. cit., p. 445-456; ClaIR, Pierre, " La vie intellectuelle à Saumur au XVII ${ }^{\mathrm{e}}$ siècle ", Marseille, $\mathrm{n}^{\circ} 101$ (1975), p. 121-124; Dray, J.-P., " The Protestant Academy of Saumur and its relations with the Oratorians of Les Ardilliers ", History of European Ideas, t. 9 (1988), p. 465-478; MAILLARD, Jacques, "L'Oratoire de Saumur et les protestants au XVII e siècle ", in LEBRUN, François, Saumur, capitale européenne du protestantisme..., op. cit., p. 125-135. J'ai pu consulter jadis aux archives de l'Oratoire de Paris le précieux dossier manuscrit de GoISNARD, A.W., L'École de Théologie de Notre-Dame-des-Ardilliers de Saumur (21+177 p.), qui a exploité toutes les sources et recense tous les élèves qu'il a repérés, ainsi que son étude également inédite Le Séminaire de l'Oratoire et l'École de Théologie aux Ardilliers (1635-1745) (ms. Saumur, Les Ardilliers, 1966). Voir pour le site : EngueHARD, Henri, "L'Église Notre-Damedes-Ardilliers et le couvent de l'Oratoire à Saumur ", Actes du Congrès archéologique de France, $122^{e}$ session, 1964 : Anjou, Paris, 1964, p. 584-597.

15. Sur l'Oratoire et la Hollande, voir FrJuHOFF, Willem, "The Oratory in the seventeenthcentury Low Countries ", Revue d'histoire ecclésiastique, t. 107, no 1 (2012), p. 169-222.

16. Voir SPIERTZ, Mathieu Gerardus, "Jeugd- en vormingsjaren van Johannes van Neercassel, apostolisch vicaris (1663-1686) ", Archief voor de geschiedenis van de katho- 


\section{Saumur, le " grand tour " et les jeunes Néerlandais}

Le séjour des étudiants étrangers à Saumur s'inscrit dans le phénomène moderne qui, avec un nom quelque peu trompeur, est souvent appelé le " grand tour ${ }^{17}$ ". Ce nom générique cache en fait différents types de voyages à l'étranger qui, de surcroît, ont changé de caractère au cours de l'Ancien Régime. Alors que le grand tour $\mathrm{du} \mathrm{XVII}^{\mathrm{e}}$ siècle concerne essentiellement un vaste public étudiant englobant l'ensemble de l'Europe de l'Ouest et recrutant dans toute la moitié septentrionale de l'Europe, de l'Irlande aux pays baltes en passant par la Grande-Bretagne, les Pays-Bas, la Scandinavie, le Saint-Empire et la Pologne, celui du XVIII ${ }^{\mathrm{e}}$ siècle est essentiellement nobiliaire, touristique ou savant, et italien, et il concerne avant tout les couches supérieures très fortunées des empires britannique et germanique, occasionnellement celles de la Hollande et des pays scandinaves, mais le plus souvent sans objectif étudiant. Les "touristes " du XVII ${ }^{\mathrm{e}}$ siècle voyageaient en groupes d'amis, de proches parents ou de camarades d'étude, ceux du $\mathrm{XVIII}^{\mathrm{e}}$ siècle se faisaient accompagner de leurs domestiques.

Pour le XVII ${ }^{\mathrm{e}}$ siècle, on peut distinguer au moins trois types de voyages accomplis par les voyageurs/étudiants :

- la pérégrination académique : le voyage de fin d'études du véritable étudiant, appelé une peregrinatio academica impliquant au minimum l'inscription dans une matricule universitaire, la participation à la vie d'une nation étudiante ou une fraternité estudiantine, ou l'acquisition d'un grade universitaire, même sans études préalables sur place;

- le voyage éducatif : celui du jeune adulte cherchant à se dépayser à l'étranger dans un but éducatif, en y ajoutant l'acquisition de connaissances ou aptitudes particulières qui lui seront utiles dans sa vie ultérieure, tels

lieke kerk in Nederland, t. 18 (1976), p. 169-197; VoORVElT, Cornelius Petrus, De Amor poenitens van Johannes van Neercassel (1626-1686). Ontstaansgeschiedenis en lotgevallen van een verhandeling over de strenge biechtpraktijk, Zeist, Kerckebosch, 1984; du même, "L'Amor poenitens de Neercassel ", in VAN BAVEL, J. et ScHRAMA, Martijn, Jansénius et le jansénisme dans les Pays-Bas. Mélanges Lucien Ceyssens, Louvain, Peeters/University Press, 1982, p. 66-92.

17. De Ridder-Symoens, Hilde, "Mobility ", in De Ridder-Symoens, H. (dir.), A History of the University in Europe. Vol. I: Universities in the Middle Ages, Cambridge etc., Cambridge University Press, 1992, p. 280-304; vol. II : Universities in Early Modern Europe (ibid., 1996), p. 416-448; FrIJHOFF, Willem, " La circulation des hommes de savoir : Pôles, institutions, flux, volumes ", in BoTs, Hans, WAQUET, Françoise (dir.), Commercium litterarium. La communication dans la République des Lettres/Forms of communication in the Republic of the Letters, 1600-1750, Amsterdam et Maarssen, APA-Holland University Press, 1994, p. 229-258; du même, "Éducation, savoir, compétence. Les transformations du Grand Tour dans les Provinces-Unies à l'époque moderne ", in BABEL, Rainer et PARAVICINI, Werner (dir.), Grand Tour: Adeliges Reisen und europäische Kultur vom 14. bis zum 18. Jahrhundert. Akten der internationalen Kolloquien in der Villa Vigoni 1999 und im Deutschen Historischen Institut Paris 2000 [Beihefte der Francia, 60], Ostfildern, Jan Thorbecke Verlag, 2005, p. 609-635; VERHOEVEn, Gerrit, Anders reizen? Evoluties in vroegmoderne reiservaringen van Hollandse en Brabantse elites (1600-1750), Hilversum, Verloren, 2009, trad. comme Europe within Reach: Netherlandish Travellers on the Grand Tour and Beyond, 1585-1750, Leyde et Boston, Brill, 2015. 
les rituels de la civilité, l'apprentissage de la langue du pays, l'équitation, l'escrime, la danse, le luth, etc. Pour le dire avec la belle formule de Thomas Erpenius (1584-1624), le professeur surdoué de langues orientales à Leyde mort précocement de la peste, qui lui-même avait passé l'année 1610 à l'académie de Saumur au cours de son grand tour par l'Angleterre, la France, l'Italie et l'Allemagne : "Finis peregrinationis sit notitia sextuplex : linguae, regionis, religionis, rerum gestarum, morum et clarorum virorum ${ }^{18}$ ";

- le voyage touristique : celui du touriste passant simplement de ville en ville pour découvrir des monuments, des antiquités, les mœurs et coutumes, souvent en attendant un moment de passage dans une université de son choix pour y prendre un grade.

Cette distinction se reflète dans les noms qui ont été donnés au voyage à l'étranger à la suite d'un débat scientifique récent dans les Pays-Bas : voyage d'études (studiereis) vers une ou plusieurs des principales universités de l'Empire, de la France et de l'Italie pour la première catégorie, voyage éducatif (educatiereis, ou en allemand Kavalierstour s'il s'agit de nobles ou aristocrates) pour la deuxième, le terme de «touriste " étant appliqué aux trois catégories ${ }^{19}$.

L'analyse des journaux de voyage des touristes néerlandais du XVII ${ }^{\mathrm{e}}$ siècle qu'Anna Frank-van Westrienen a menée dans sa thèse sur le grand tour montre bien comment la tension, puis la séparation, entre l'objectif savant et l'objectif social ont finalement conduit à un clivage entre le grand et le petit tour. Le voyage de la majorité évolue vers un grand tour hybride, surtout caractéristique des Néerlandais en raison des besoins et possibilités de leur milieu d'origine essentiellement bourgeois et de leur destinée sociale, culturelle et politique aux leviers de commande des villes et provinces de leur nouvel État. Par ailleurs, l'accent qui à la fin du XvI ${ }^{\mathrm{e}}$ et au début du XVII ${ }^{\mathrm{e}}$ siècle était mis parmi les Néerlandais sur la recherche d'académies et universités réformées, de doctrine éprouvée - telles que Steinfurt, Hamm, Herborn, Marbourg, Heidelberg, Bâle, Lausanne, Genève, Sedan ou Saumur - disparaît au cours de la première moitié du XVII ${ }^{\mathrm{e}}$ siècle. Le voyage éducatif se déconfessionnalise alors rapidement, même si la pérégrination académique au sens strict continue d'être importante pour certains, surtout pour les candidats au saint ministère qui, financièrement démunis, jouissent parfois de bourses d'études provinciales ou locales pour l'étranger les obligeant à choisir des universités théologiquement sûres. D'autre part, comme l'a très justement fait remarquer Paul Dibon,

18. ERPENIUS, Thomas, De peregrinatione Gallica utiliter instituenda tractatus, Leyde, Franciscus Hegerus, 1631, p. 2. Notice biographique : Nieuw Nederlandsch Biografisch Woordenboek, t. VIII (1930), col. 495-496; DiBon, Paul, " Le voyage... ", art. cit., p. 10.

19. Frank-VAn Westrienen, Anna, De Groote Tour. Tekening van de educatiereis der Nederlanders in de zeventiende eeuw, Amsterdam, Noord-Hollandsche Uitgeversmaatschappij, 1983; BoTs, Hans et FRIJHOFF, Willem, "Pérégrination académique ou voyage éducatif? Remarques à propos des voyageurs du Brabant septentrional : nature, fréquence et circuits de leurs voyages sous l'Ancien Régime ", in KULCZYKOWSKI, Mariusz (dir.), Pérégrinations académiques. Ive session scientifique internationale, Cracovie, 19-21 mai 1983 [Prace historyczne, 88], Cracovie, Éditions de l'université Jagellone, 1989, p. 117-129. 
la pérégrination académique se rétrécit et se déplace vers le pays modèle de la nouvelle civilité, des bonnes manières et de la culture de l'élite : la France. Dans cet iter gallicum, Saumur prend désormais la place qui avait auparavant été réservée à Heidelberg et Genève comme hauts-lieux du protestantisme réformé.

Vers 1650, le voyage type réunit et réconcilie donc au moins quatre exigences :

- se faire éduquer à un standard européen de conduite dans les hauts lieux de l'étiquette nouvelle pour apprendre la civilité et assumer le modèle de la société de cour incarnée par la France;

- prendre un grade universitaire en droit ou en médecine (peu importe l'endroit, mais Orléans pour le droit et Angers ou Caen pour la médecine dominent nettement);

- prendre contact avec des savoirs anciens, universellement reconnus, dans lesquels on peut s'enraciner face à la science internationale, ou encore avec un savoir autre, que l'on ne trouve pas chez soi;

- enfin, acquérir des compétences sociales et se constituer un réseau d'amis, de relations, de patrons ou de clients.

Le transfert culturel que le grand tour opère ainsi dans la vie du « touriste " conduit à terme à un vrai changement culturel dans sa propre vie et son propre réseau, par le biais des formes d'appropriation des valeurs et conduites culturelles que le jeune homme sait désormais mettre en œuvre et dont, d'ailleurs, les modalités lui sont enseignées au cours même de son voyage par ses compagnons.

Dans tous les cas, le voyage est accompli en compagnie d'autres jeunes, soit étudiants soit commis de marchands ou apprentis, parfois accompagnés d'un domestique ou d'un précepteur. Le séjour lui-même peut s'étaler sur plusieurs années. Ces deux caractéristiques assurent à cette élite aisée, souvent même fortunée, et promise à des charges importantes dans l'État, une cohésion qui dure toute la vie. Le rituel du voyage était donc extrêmement important pour la formation des futures élites dirigeantes. Les jeunes en étaient eux-mêmes aussi conscients que leurs parents. Ils consacraient beaucoup de temps à toutes les formes de socialisation, y compris par le jeu et les excursions de groupe, et ils inscrivaient leurs nouvelles relations dans les livres d'amis, des albums d'armoiries, des correspondances, des collections de poèmes dédiés à leurs compagnons, etc. Dans cette perspective, l'objectif concret du voyage importait moins que le fait même de l'accomplir, même si l'on y reconnaît bien un certain nombre d'itinérairestype, englobant les sites royaux d'Angleterre et de France, les monuments incontournables (tel le pont du Gard), les principales villes universitaires (Orléans, Bourges, Poitiers, Angers, Caen), les hauts-lieux du protestantisme (La Rochelle), et les lieux de villégiature les plus agréables. Parmi ces derniers, on citera donc en premier lieu Saumur, où la visite de l'académie paraît bien souvent un prétexte commode pour un séjour de formation générale, de visites ciblées et de détente prolongée. 
Au cours du XVII ${ }^{\mathrm{e}}$ siècle, on voit naître plusieurs types de circuits de substitution à l'ancien grand tour germano-franco-italien, trop long, trop cher et aussi trop dangereux en raison des mouvements de guerre, des périls de la mer, ou des dangers de la route sur le parcours italien. Au cours de la première moitié du XVII ${ }^{\mathrm{e}}$ siècle, du fait même de la longue guerre hispano-néerlandaise et de la Guerre de Trente Ans dans l'Empire, le grand tour se réduit déjà insensiblement au royaume de France. Mais le nouveau circuit privilégié par le plus grand nombre est le " petit tour " intra-français, réduit au circuit de la Loire dans un grand quadrilatère limité par Paris, Caen, Nantes, Poitiers et Bourges, avec Orléans et Angers comme épicentres, le tout précédé ou suivi d'une excursion à la cour de Londres et dans les villes universitaires d'Oxford ou Cambridge. Ce " petit tour " rapidement connu comme tel avait l'avantage de pouvoir atteindre tous les objectifs, tout en étant moins cher, moins long et plus sûr que le passage par l'Italie ou l'Allemagne ou la traversée des Alpes.

Vers 1600, la fondation de l'académie de Saumur vient à point nommé pour faire jouer à la ville un rôle central, parfois prépondérant, dans le tourisme des jeunes néerlandais, par son site avantageux, son rôle de villégiature, les attraits de la vie locale dans une ville petite au climat doux et au vin agréable à boire, et son caractère de haut-lieu et de refuge réformé dans un univers catholique ${ }^{20}$. Nous savons par leurs journaux ou lettres et par de nombreuses références dans d'autres sources que Saumur était une ville de passage quasi obligée pour l'ensemble des jeunes voyageurs néerlandais réformés, qu'ils soient de vrais étudiants en théologie ou des proposants, tels le botaniste Adolphus Vorstius (1597-1663) ou le cartésien Louis Wolzogen (1633-1690), des jeunes en quête d'une éducation de qualité, ou de simples passants. Toutefois, le choix restait sujet à des préférences personnelles. Ainsi, le futur philologue Johannes Fridericus Gronovius (1611-1671) qui, en 1640, fait son grand tour en tant que précepteur des richissimes frères De Geer, délaisse Saumur à dessein, préférant Angers, où il prend son grade en droit. La colonie hollandaise y était encore plus nombreuse et davantage à son goût ${ }^{21}$.

D'autres jeunes étrangers, en revanche, faisaient le choix délibéré de Saumur comme lieu d'études, même s'ils n'avaient aucune velléité théologique. Cela vaut pour Johannes Hudde (1628-1704), futur puissant bourgmestre d'Amsterdam, féru d'innovations techniques, lui-même fils d'un grand négociant amstellodamois, directeur de la compagnie de commerce

20. Sur les étrangers à Saumur : BouRCHEnin, Pierre Daniel, Étude sur les académies protestantes..., op. cit., p. 402-404; JOUBERT, André, "Les étudiants allemands de l'Académie protestante de Saumur et leur maître de danse (1625-1642) d'après un document inédit ", Revue d'Anjou, t. 18 (1889), p. 158-161; différents noms de touristes à Saumur chez DiBon, Paul, «Le voyage... ", art. cit., p. 119-120, 130-135, 140, 145-150.

21. Dibon, Paul et Waquet, Françoise, Johannes Fridericus Gronovius, pèlerin de la République des Lettres, Genève, Droz, 1984, p. 7. Louis de Geer figure dans l'album du maître de danse Du Puy-Rideau : JouBERT, André, "Les étudiants allemands... ", art. cit., p. 161 . 
du Levant et de la compagnie des Indes Orientales. Pendant ses études de philosophie et de droit à Leyde, il commence à s'intéresser aux mathématiques, qui continuent de l'occuper pendant de longues années. Son grand tour en compagnie du jeune candidat en médecine Hendrik van Heuraet (1634-1660?) le conduit en juin 1658 à Saumur, où les deux compères séjournent probablement une année entière ${ }^{22}$. Van Heuraet retourne ensuite à Leyde où il s'inscrit de nouveau en médecine le 7 novembre 1659, mais on perd sa trace peu après. Ce voyage de Saumur est à mettre en rapport avec l'intérêt que les jeunes Néerlandais passionnés des sciences nouvelles portent au mathématicien Melchisédech Thévenot (ca. 1620-1692) et aux réunions hebdomadaires de son académie domestique à Paris, car on retrouve cette combinaison dans d'autres curricula, tel celui de l'Amstellodamois Jan Swammerdam (1637-1680), candidat en médecine et futur insectologue célèbre qui, au printemps 1664, séjourne quelques mois à Saumur pour suivre les cours de Tanneguy Le Fèvre (1615-1672) et discuter avec Isaac d'Huisseau (1607-1672), avant de rejoindre en septembre de cette même année, à Paris, Thévenot, chez qui il restera une année entière en compagnie de son ami danois Niels Stensen (Steno, 1638-1686) ${ }^{23}$.

\section{Passants à Saumur}

S’il n'y a plus de registres matricules des étudiants de Saumur, le passage à l'académie ou dans la ville est mentionné dans de très nombreux journaux de voyage ou alba amicorum (livres d'amis) ${ }^{24}$. Certaines correspondances nous renseignent sur le concret de la vie, parfois amplement, comme celle du précepteur des frères Kerr séjournant à Saumur en 1654, qui a été publiée sur l'Internet par Jean-Paul Pittion ${ }^{25}$. Les frères Kerr faisaient partie de l'aristocratie écossaise qui s'était engagée dans l'armée de la République des Provinces-Unies et leur grand tour débuta à Leyde.

22. Sur Van Heuraet : Nieuw Nederlandsch Biografisch Woordenboek, t. I, Leyde, Sijthoff, 1911, col. 1098-1099; VAN MAANEN, Jan A., " Hendrick van Heuraet (1634-1660?), his life and mathematical work ", Centaurus, t. 27 (1984), p. 218-279. Son Epistola de curvarum linearum in rectas transmutatione fut plus tard ajoutée aux éditions de la Geometria de Descartes. Sur Hudde : Nieuw Nederlandsch Biografisch Woordenboek, t. I, Leyde, Sijthoff, 1911, col. 1172-1176.

23. VAN BerKel, Klaas, "Swammerdam, Leeuwenhoek et la science française ", Septentrion, t. 13 (1984), p. 58-63; BAAR, Mirjam de, " Hartsemblematiek in Swammerdams studie van de eendagsvlieg ", De Zeventiende Eeuw, t. 21 (2005), p. 312-334; KoolJMANs, Luuc, Gevaarlijke kennis : inzicht en angst in de dagen van Jan Swammerdam, Amsterdam, Bert Bakker, 2007.

24. Cf. sur ces sources : DEKKER, R.M., "Dutch travel journals from the sixteenth to the early nineteenth centuries ", Lias. Sources and documents relating to the early modern history of ideas, t. 22 (1995), p. 277-279; ainsi que la mise au point amplement annotée de BoEdeker, Hans Erich, "Sehen, hören, sammeln und schreiben. Gelehrte Reisen im Kommunikationssystem der Gelehrtenrepublik ", Paedagogica Historica, t. 38 (2002), p. $505-532$.

25. [http://archives.ville-saumur.fr/am_saumur/app/03_archives_en_ligne/01_academie_protestante/chapitre4/Séjourner à Saumur.pdf]. 
Quant aux simples "passants ", prenons comme exemple le jeune Everhard Avercamp (vers 1595-1666), fils du pharmacien ordinaire de la ville de Kampen dans la province d'Overyssel et frère du peintre de genre Hendrick Avercamp, célèbre pour ses scènes de patinage; en 1640 il devient lui-même médecin ordinaire de Kampen ${ }^{26}$. Suivant les données de son album, il s'embarque le 16 août 1619 à Rotterdam pour Nantes, remonte la Loire jusqu'à Angers où il reste au moins quatre mois faisant des excursions plus ou moins prolongées à Thouarcé, Loudun, Saumur et SaintMathurin-sur-Loire. Par Orléans il rejoint Paris puis repart pour Valence, Orange (où il reste deux ans), Montpellier et les antiquités romaines du Midi avant de remonter peu avant décembre 1622 vers Angers pour un nouveau séjour angevin de six mois. Enfin, il traverse la Loire aux Pontsde-Cé pour regagner Thouarcé, puis Nantes d'où il rentre aux Pays-Bas. Le 15 décembre 1620 il est à Saumur, où pendant une semaine pas moins de douze étrangers, futurs médecins, juristes ou magistrats, apportent une dédicace dans son album : Joannes a Velsen de Leeuwarden, Albertus van Loo de Dordrecht, Nicolaus Busch de Groningue, Ewald Graswinckel de Delft, le gentilhomme Bernhardus Bentinck de l'Overyssel, Petrus et Theodoricus Albada de Groningue, Aegidius Godefridi van Blanckendael de Zélande, Cornelius Dosthorne et les Écossais Jacobus Peirsoune, R. Gourlaijus, et David Lermonthus, enfin Johannes Gerhardi de Dieppe - en somme, des représentants de quasiment toutes les provinces néerlandaises et des deux grandes nations réformées, l'Écosse et la France!

Un autre exemple est l'album de Johan Kaldenbach (vers 1583-1636), fils aîné d'Adam, procureur ordinaire de la ville de Zutphen, puis secrétaire de justice, depuis 1593 échevin et bourgmestre, et dès 1609 membre du Conseil d'État de la République. Cet album permet de reconstruire le grand tour qu'il a entrepris entre 1607 et 1609 et de reconnaître son passage à Saumur. Il s'agit d'une forme mixte de voyage d'études et d'éducation à la suite de ses études de droit, accomplies depuis 1599 à Franeker, à l'académie de Steinfurt et à Leyde. Passant successivement par l'université de Marbourg, l'académie des nobles à Cassel, l'université de Heidelberg, les académies de Lausanne et Genève, il se rend à Orléans où, en 1608, il devient docteur en droit en étant assesseur de la nation germanique, puis à Angers et Saumur où il a dû séjourner plusieurs semaines. Il gagne ensuite Orléans pour un second séjour comme senior de la nation, puis Paris et enfin, à nouveau, l'université de Leyde. Rentré chez lui en Gueldre il devient avocat, échevin en 1616 et bourgmestre de Zutphen, pour finir en 1620 conseiller gradué à la Cour de Gueldre ${ }^{27}$.

26. Notice biographique dans Nieuw Nederlandsch Biografisch Woordenboek, t. X, Leyde, Sijthoff, 1937, col. 27-28; NANNINGA UITTERDIJK, Jurgen, " Het Album Amicorum van Dr. Everhardus Avercamp, 1619 ", Bijdragen voor de geschiedenis van Overijssel, t. 6 (1880), p. 219-264; DiBon, Paul, « Le voyage... ", art. cit., p. 134-135 (erreur de date).

27. FrIJHOFF, Willem, « De "grote tour" van een Zutphens burgerzoon : Johan Kaldenbach en zijn album amicorum (1604-1609) ", Oud-Zutphen. Tijdschrift van de Historische Vereniging Zutphen, t. 3 (1984), p. 38-54. 
Quatre ans après le séjour de Kaldenbach, le 31 mars 1613, Cornelis van Beveren (1591-1663), fils d'un puissant bourgmestre de Dordrecht, la première ville de Hollande, et lui-même promis à la régence de sa cité, visite Saumur au pas de course au cours de son grand tour, passant par la France, la Suisse et l'Allemagne. Avant de visiter à la même vitesse Angers et le collège jésuite de La Flèche, qui le remplit d'admiration, il passe un jour et une nuit à Saumur pour visiter le château et le Collegium Theologiae et Philosophiae. Malgré la précipitation dont il fait preuve, il réussit tout de même à rendre visite au gouverneur Du Plessis-Mornay, qu'il décrit dans ces termes : "Monsieur du Plessis est un homme savant et agréable, d'une belle stature, blanc, les yeux à fleur de tête, qui passe le plus fort de son temps à étudier ${ }^{28}$."

D'autres étudiants suivent approximativement le même itinéraire stéréotypé, en particulier les très nombreux passants qui visitent Saumur à partir d'un autre point de chute, le plus souvent Angers et son université célèbre. Angers est alors une ville très appréciée où de très nombreux Néerlandais séjournent et où nombre d'entre eux obtiennent un diplôme en droit ou en médecine ${ }^{29}$. Les étapes obligatoires autour de Saumur sont l'amphithéâtre antique de Doué, la ville de Loudun, l'abbaye de Fontevraud et le collège de La Flèche, où les Néerlandais font avidement la connaissance de la grande entreprise éducative des jésuites qui apparemment les reçoivent avec courtoisie et intérêt. Mais au sein même de ces grappes de "touristes " qui ne sont que de simples passants, l'ennui peut frapper, comme le montre cette citation du journal de voyage de deux gentilshommes natifs de La Haye, les frères Zoete de Laecke, qui fréquentent surtout les académies d'équitation. Le 12 février 1657, ils vont voir dans son domicile à Paris, rue aux Ours, Philibert van Tuyl, seigneur de Serooskerke (mort en 1661), un gentilhomme zélandais haut placé, " qui après avoir fait le tour de France, sans y avoir rien appris ny remarqué que les maisons et les rües des villes, retourna au commencement de l'automne à Saumur, où un jour traittant quelques-uns de ses amis, il les fist tant boire, qu'il y eut un d'Amsterdam qui tua un bourgeois ${ }^{30}$ ".

28. Bevere, Cornelis de, Notulen, conservé au Centraal Bureau voor Genealogie (La Haye), Familiearchief Beelaerts van Emmichoven, ms. 119. Sur Du Plessis-Mornay : PATRY, Raoul, Philippe Du Plessis-Mornay, un huguenot homme d'État (1549-1623), Paris, Fischbacher, 1933; РотоN, Didier, « Réforme et guerres de religion... ", art. cit., p. 150-151, pour ses relations précoces avec les Pays-Bas.

29. FRIJHOFF, Willem, "Étudiants étrangers à l'Académie d'équitation d'Angers au $\mathrm{XVII}^{\mathrm{e}}$ siècle ", Lias. Sources and documents relating to the early modern history of ideas, t. 4, no 1 (1977), p. 13-84; FrIJHOFF, Willem et VAN DRIE, Rob, " Het wapenboek van de Nederlandse studentenvereniging te Angers, 1614-1617 ", Jaarboek van het Centraal Bureau voor Genealogie en het Iconographisch Bureau, t. 44 (1990), p. 115-148; FrIJHOFF, Willem, "Nederlandse promoties in de geneeskunde aan Franse universiteiten (zestiende-achttiende eeuw) ", Jaarboek van het Centraal Bureau voor Genealogie, t. 60 (2006), p. 75-112.

30. FAUGĖRE, Armand-Prosper (publ.), Journal du voyage de deux jeunes Hollandais à Paris en 1656-1658, Paris, Champion, 1899, p. 76. 
Arnout Hellemans Hooft (1629-1680), fils de l'Amstellodamois Pieter Cornelisz Hooft, bailli de Muiden et un historien et littérateur célèbre dans le paysage culturel néerlandais, fait son grand tour en 1649-1651. Il change quasiment tous les jours de ville, et ses observations d'une brièveté et d'une banalité déconcertantes sont assez caractéristiques de la plupart des journaux de voyage. Le 22 août 1651, il quitte Nantes pour Clisson et Mortagne, le 23 il est à Thouars, le 24 à Loudun, le 25 à Richelieu, le 26 à Montreuil-Bellay puis Doué où l'amphithéâtre le déçoit et Saumur où il loge dans l'auberge " À la Corne [de Cerf] ", à sa satisfaction. Le lendemain 27, il y va à l'église, pleine de gens, et visite le château doté de belles murailles et entouré d'eau; il y rend visite à Monsieur et Mademoiselle Niot. Mais le 28 il est déjà à Tours. Il rencontre continuellement des compatriotes en route et, partout où il va, il cherche leur compagnie. Le dépaysement semble assez limité pour ce jeune homme, mais il est vrai qu'il finit en Anjou son voyage de deux ans à travers l'Allemagne, la Suisse, l'Italie et la France et qu'après tous ces dépaysements bien peu de choses arrivaient encore à l'enthousiasmer ${ }^{31}$.

Si beaucoup de visiteurs sont donc passés par Saumur, quelques-uns seulement ont laissé des journaux de voyage assez détaillés pour permettre de découvrir quelles étaient exactement leurs occupations et leurs impressions de la ville, sans même parler de leur vie de tous les jours et de l'enseignement à l'académie. Jacob Olfertsz de Jong (1599-1646), par exemple, le second fils d'Olfert Barentsz, bourgmestre de Hoorn en Westfrise, mentionne dans son journal avec précision les étapes de son voyage du $1^{\mathrm{er}}$ avril 1621 au 7 août 1622. Celui-ci ne dépasse pas le circuit du " petit tour " par Rouen, Paris, Orléans, Angers, Caen et Dieppe. Il se déplace le plus souvent en compagnie de son frère aîné Jan et de concitoyens qu'il rencontre en route, car les itinéraires des touristes se croisaient souvent et on apprend dans les journaux de voyages qu'ils communiquaient activement entre eux, ne serait-ce que par précaution face aux dangers de toutes sortes qui les menaçaient. Le 11 décembre 1621, il prend le bateau à Blois pour arriver deux jours plus tard à Saumur où il trouve un logement chez un monsieur La Moche, avec son frère qui le quitte cependant quelques jours plus tard pour rentrer chez lui. Tout ce que nous apprenons de son séjour de trois mois et demi à Saumur est son excursion à l'amphithéâtre de Doué et dans quelques autres "petites villes " non spécifiées. Cependant, pendant ce séjour il tombe gravement malade. Son hôte, le médecin et l'apothicaire cherchent à tour de rôle à profiter de sa maladie (ils le " rasaient ", comme il écrit), de sorte que ses moyens financiers s'épuisent rapidement. Aussi se fait-il transporter à Bourgueil où, le 26 mars 1622, il trouve pour un prix plus modéré le gîte chez monsieur Olivier à la Fontaine. Il y reste trois mois pour se rétablir, jusqu'au 19 juin 1622. Contrairement aux Saumurois, qu'il décrit

31. Hooft, Arnout Hellemans, Een naekt beeldt op een marmore matras seer schoon. Het dagboek van een " grand tour " (1649-1651), publ. par GRABOWSKY, E.M. et VERKRUIJSSE, Pieter Jozias, Hilversum, Verloren, 2001, p. 180-181. 
comme rapaces, les habitants de Bourgueil, son hôte compris, sont courtois et accueillants. Qui plus est, son français s'y améliore à vue d'œil, étant donné l'absence d'étrangers, à l'exception d'un unique Néerlandais de passage, Otto Valck de Groningue, ce qui du coup lui donne une belle occasion de se débaucher. À Bourgueil, il fournit lui-même l'hospitalité à Monsieur d'Allères, seigneur de Bonnétable, lorsque celui-ci y courtise une femme du cru. Ailleurs en France, on traite notre Jacob Olfertsz de " parpaillot " - nous sommes dans les années critiques de l'offensive antiprotestante. Au Havre il est suspecté d'espionnage et menacé d'emprisonnement, en dépit de son passeport d'étudiant délivré par la Nation Germanique d'Orléans, et en fin de compte il y achète un cheval pour se précipiter à Dieppe où le duc de Longueville lui permet de rentrer chez lui, bien soulagé ${ }^{32}$.

\section{Joan Huydecoper, un jeune aristocrate en formation}

Deux voyageurs se détachent clairement du lot, et leurs notes de voyage permettent de souligner les deux fonctions principales de Saumur pour les jeunes étrangers. Pieter de la Court et Joan Huydecoper sont tous deux des figures clé de l'histoire de la province de Hollande, voire de la République des Provinces-Unies, de la Court en tant qu'intellectuel et auteur politique, Huydecoper en tant qu'acteur politique. Ils correspondent aussi aux deux types de voyageurs précédemment évoqués : Pieter de la Court cherche à Saumur une éducation théologique ou du moins un supplément sérieux de formation dans ce domaine, Joan Huydecoper y passe une bonne partie de son voyage de type éducatif. Commençons par ce dernier.

Joan Huydecoper appartenait à une famille qui, grâce à ses riches archives parfaitement conservées, est au cœur de deux études historiques importantes, parues presque simultanément il y a une quinzaine d'années : une étude des réseaux de parenté (dans les termes de l'époque, de la vriendschap, l'amitié), et une autre sur les pratiques éducatives de l'élite bourgeoise de la Hollande ${ }^{33}$. Selon Benjamin Roberts, l'auteur de ce dernier travail, la famille Huydecoper "symbolized the prosperity of Amsterdam during the Dutch Golden Age. Huydecoper managed to unify wealth, politics, and the cultural elite status in Amsterdam ${ }^{34}$ ".

Joan Huydecoper le jeune était né à Amsterdam le 21 février 1625. Après avoir été maintes fois bourgmestre d'Amsterdam et membre du conseil d'administration (directeur) des compagnies de commerce et de l'Amirauté, il y mourut le $1^{\text {er }}$ décembre 1704 , au sommet de l'élite de ce qui était

32. PAuW VAN WIELDRECHT, "Album amicorum van Jacob Olfertsz de Jong », De Navorscher, t. 53 (1903), p. 658-692; « Levensschets van den Westfries Jacob Olfertsz de Jongh 15991646 ", West-Friesland's Oud en Nieuw, t. 14 (1940), p. 145-159.

33. KoolJmans, Luuc, Vriendschap en de kunst van het overleven in de zeventiende en achttiende eeuw, Amsterdam, Bert Bakker, 1997, p. 113-131; RoBERTs, Benjamin, Through the keyhole. Dutch child-rearing practices in the $17^{\text {th }}$ and $18^{\text {th }}$ century: Three urban elite families, Hilversum, Verloren, 1998, p. 50-54.

34. RoBerTs, Benjamin, Through the keyhole..., op. cit., p. 50. 
encore la capitale mondiale du commerce et de la finance. Il était le fils aîné de Joan Huydecoper l'aîné (1599-1661) et de Maria Coymans ${ }^{35}$. Son père était lui-même le seul fils survivant issu du mariage de Jan Jacobsz Bal dit Huydecoper (1541/42-1624), un tanneur et marchand pelletier, et de surcroît un calviniste sévère et politicien rusé qui s'était enrichi dans le commerce du sel et, plus discrètement, dans les spéculations financières et foncières qui accompagnèrent la croissance rapide d'Amsterdam dans le dernier quart du $\mathrm{XvI}^{\mathrm{e}}$ siècle. Il était directeur de la compagnie Magellan pour le commerce sur l'Amérique du Sud. Son fils Joan l'aîné, bénéficiant d'une des fortunes les plus considérables de la ville, allait jouer un rôle actif de mécène des arts et des lettres. Échevin dès 1629, il entre en 1630 au conseil municipal avant de se faire élire bourgmestre en 1651. Adorant jouer le grand seigneur, ce calviniste très modéré rejoint rapidement la faction libérale, ce qui lui permet de se faire réélire de nombreuses fois et de représenter la ville au couronnement de Charles II d'Angleterre en 1660. Joignant à son patrimoine déjà non négligeable la fortune colossale de sa femme Maria Coymans, fille du plus important banquier d'Amsterdam à cette époque, il fait construire vers 1640 à Maarssen, du côté d'Utrecht, une superbe maison de campagne appelée Goudestein (Maison Dorée) et s'achète la grande propriété de Maarsseveen qui, fait exceptionnel dans la République, est élevée à son intention en seigneurie par les États d'Utrecht. Dès lors il s'intitule seigneur de Maarsseveen, comme son fils le faisait déjà à Saumur, pendant son grand tour. Ses prétentions aristocratiques se renforcent encore lorsque le roi de France l'admet en 1650 dans l'Ordre de Saint-Michel.

Le réalisme politique caractérise tout autant la carrière de son fils Joan le jeune. Au début de la guerre de Hollande il troque sa veste de républicain contre un habit d'orangiste, ce qui lui vaut une première élection comme bourgmestre en 1673. Son père Joan l'aîné étant non seulement extrêmement ambitieux mais aussi un éducateur très tatillon et très près de ses sous, la correspondance entre le père à Amsterdam et le fils à Saumur s'avère une longue litanie de plaintes paternelles sur les dépenses de son rejeton et ses demandes d'argent, par ailleurs justifiées par une comptabilité très précise dont l'historien ne peut que lui être reconnaissant. Le séjour de Joan le jeune à Saumur s'inscrit dans un vrai grand tour à travers toute la France ${ }^{36}$. Cependant, alors que l'essentiel du voyage est fait au pas de course, Saumur apparaît comme une étape de longue durée car il est forcé d'y attendre l'autorisation paternelle pour se rendre en Italie; son père rechigne devant les dépenses qu'il trouve trop considérables et il est visiblement effrayé par les nouvelles sur la guerre civile, la Fronde. Mais

35. EliAS, J.E., De vroedschap van Amsterdam 1578-1795, Haarlem, Loosjes, 1903-1905; reprint Amsterdam, N. Israel, 1965, t. I, p. 384-391, 518-519.

36. DiBon, Paul, "Le voyage... ", art. cit., p. 131-132, 142, 145-150. Les documents originaux se trouvent dans Het Utrechts Archief, Familiearchief Huydecoper (fonds $n^{\circ} 67$ ), dossiers $\mathrm{n}^{\circ} 53$ (registres des lettres et comptes, 1648), 66-68 (journaux de voyage, 1648-1649). 
par un chantage affectif habile, mettant à contribution ses frère et sœur et d'autres parents en Hollande, par ses insistances répétées invoquant en particulier le séjour de ses trois cousins Deutz à Rome, et par des preuves tangibles de son bon sens financier, notamment en vendant ses deux chevaux, un frison et un espagnol, à un prix assez élevé, et enfin en promettant de se contenter de 1500 florins pour une année entière (trois à quatre fois le revenu annuel d'un artisan), Joan arrive à vaincre les réticences de son père. Le 24 avril il lui écrit son " extrême joie " d'avoir obtenu la permission de partir pour Orléans, Lyon, Marseille, Gênes, Venise et finalement Rome; il ira jusqu'à Arles, mais devra malheureusement repartir pour Genève puis regagner la Hollande avant même d'avoir accosté en Italie. Quant à Saumur, arrivé dans la ville le $1^{\text {er }}$ août 1648 , il en repart finalement neuf mois plus tard, le 28 avril 1649.

Joan ne voyage pas seul, mais en compagnie de son cousin issu de germain Dirck Schaep, fils d'une nièce de Joan Huydecoper l'aîné, Lijsbeth Kieft. Par ailleurs, il a à son service un domestique, appelé Eustache, probablement un Français engagé en route, car il le quitte lors de l'annonce de son passage en Italie. Il lui paie 20 florins $^{37}$ par mois, l'entretien de ses habits et ses frais médicaux. Joan et Dirck se séparent à leur départ de Saumur après y avoir rencontré quantité d'autres Néerlandais faisant le grand tour comme eux. Dirck était un homme ambitieux qui allait être secrétaire de la ville d'Amsterdam de 1655 à 1697 et servir pendant la guerre de Hollande (en 1673-1675) comme ambassadeur en Suède, où il fut anobli le 18 novembre 1674. De l'avis de Joan Huydecoper, Dirck était pingre, mais le registre des dépenses de Joan montre qu'il ne savait pas bien gérer ses finances lui-même et que Dirck dut lui avancer de l'argent à plusieurs reprises. Par ailleurs, le registre fourmille de petites dépenses pour les étrennes, les pauvres, les bonnes, les domestiques, les valets d'écurie et les messagers, les apprentis de toutes sortes, les garçons postiers et les musiciens de passage, qui nous font entrer de plain-pied dans la vie quotidienne du riche touriste mis à contribution par les gens du peuple.

Ce registre contient également les copies des lettres qu'il a envoyées et reçues, et qui permettent de reconstruire tout son itinéraire. Il part de Genève le $1^{\mathrm{er}}$ mai 1648 , est à Montpellier le 18 mai, à Toulouse le 26, à Bordeaux le 3 juin, à Saintes le 10 juin, puis il passe par La Rochelle, par Nantes où il se loge chez le marchand hollandais Van Rhijn, et par Angers, pour enfin arriver à Saumur. Dès son arrivée il écrit une lettre en latin à son père : "Urbem hanc satis iucundam invenio. [...] Quoad linguam Gallicam, exercitiaque omnia hic optime addiscuntur ", une ville agréable, parfaite pour apprendre le français et les exercices mondains de son état. Il loge d'abord chez le maître tailleur Gotiers, à qui il achète un habit pour tous les jours (53 florins) et un habit de luxe (près de 139 florins - soit pas moins de la moitié du revenu annuel d'un artisan hollandais) et où, pour 8 sous par

37. L'unité monétaire utilisée par Joan dans ses comptes n'est pas toujours claire. À défaut, j'adopte le florin dans cette analyse. 
jour, il peut également mettre ses deux chevaux à l'écurie, le cheval frison de son domestique et le sien propre, un cheval espagnol apparemment racé, dont il prend beaucoup de soin et dont la valeur est près du triple de son cheval frison. En mars 1649, il les vend pour respectivement 30 et 80 pistoles (1 100 florins au total) au marquis de Chassé, probablement par l'intermédiaire du négociant hollandais Van Rhijn de Nantes. Il a déjà repéré deux maîtres d'équitation dans la ville de Saumur et cherche encore un précepteur de français. Le tabac hollandais lui manque; aussi écrit-t-il le même jour à son fournisseur Van de Sande en Hollande en demandant de lui envoyer une boîte contenant des pipes et du tabac par l'intermédiaire du marchand Cannars. Un mois et demi plus tard, le 14 septembre, le colis arrive enfin. Si Van de Sande est son relais pour les achats hollandais, Cannars est son contact saumurois régulier avec la Hollande, il agit comme son banquier. Il existait d'ailleurs une petite communauté marchande néerlandaise à Saumur, originaire aussi bien d'Amsterdam que de la ville d'Utrecht, à en juger d'après les noms de famille (Van Rossum, Van Wijckersloot, Van Velthuysen, Van Bredenberg, etc.) ${ }^{38}$.

Ce n'est pas l'académie qui constitue l'objectif de son séjour à Saumur, mais la ville elle-même, appréciée pour sa douceur de vivre, la découverte du pays, de ses habitants et de ses coutumes, et les exercices mondains du parfait gentilhomme dont il parlait déjà dans la première lettre à son père. Le gros des dépenses concerne la pension ordinaire, soit 529 florins au total pour les neuf mois. Il les paie par mois à ses logeurs, le premier mois au tailleur Gotiers, puis à Madame Pierson; il faut y ajouter ses dépenses personnelles " extraordinaires " (152 florins) et la dépense nécessaire pour le soin et le logement de ses deux chevaux. Les comptes mentionnent aussi l'achat de quelques spécialités : une paire de perdrix, des melons, plusieurs pots de vin qu'il achète au Lion d'Or, trois boîtes de confitures, des raisins et pistaches, deux fromages, et des huîtres que lui procure la bonne du barbier. La visite des environs de la ville prend une place de choix dans ses dépenses et sa correspondance. Ainsi, du 9 au 25 octobre il part avec son cousin en excursion pour visiter au pas de course Fontevraud, Chinon, Richelieu, Châtellerault, Poitiers, Loudun, Thouars, Doué, Brissac, Angers, La Flèche et Baugé. Ils prennent des guides sur place et se font recevoir dans les châteaux sur leur passage. Ce voyage lui coûtera plus de 110 florins. De retour à Saumur, il se plaint de l'automne pourri qui l'oblige à rester à la maison, et de ses compatriotes hollandais qui restent toujours entre eux et "summopere tabaco deditos".

Il aspire à partir pour Orléans " considérant l'accademie des estudes que l'on exerce, e[s]t la plus fameuse qui soit dedans toutes les villes où j'aije passé ", mais il a dû s'intéresser avant tout à la vie sociale de la Nation Germanique d'Orléans, car il avait déjà fini ses études par un grade en droit pris dès 1646 à Leyde. Ce geste rendait son grand tour d'ailleurs atypique

38. ChAREYRE, Philippe, "Les protestants de Saumur au XVII ${ }^{\mathrm{e}}$ siècle : religion et société ", in LEBRUN, François, Saumur capitale européenne du protestantisme..., op. cit., p. 53-62. 
car la quasi-totalité des étudiants prenaient leur grade en route dans une université française, à moindres frais, en tant que candidat "forain ", non autorisé à pratiquer le droit ou la médecine en France même. L'académie protestante de Saumur, quant à elle, ne figure dans les cahiers de ce calviniste qu'au détour d'une phrase. On apprend juste qu'il a payé 12 florins au relieur (sans doute pour des thèses ou d'autres ouvrages reçus de ses compatriotes ou des professeurs) et des sommes minimes mais régulières pour papier, plumes et crayons. Par contre, ce protestant convaincu s'extasie devant le "superbum ultimumque collegium Patrum Oratorum Nostre Dame d'Ardilliers ", sans cependant remarquer les rituels catholiques qui y sont accomplis ou s'en scandaliser, comme l'avait fait quelques années auparavant Pieter de la Court. Il achète pour 5 florins un dessin de Saumur et se fait faire pour 15 florins son propre portrait.

Dans toutes les relations de voyage, les exercices dans les académies mondaines et les leçons auprès de maîtres particuliers forment une part substantielle des occupations et dépenses des touristes. Ce sont autant d'instances de sociabilité commune de la faune touristique étrangère. C'est dans ces académies d'équitation, d'escrime, de danse ou de musique, occasionnellement aussi des cercles littéraires, que les Allemands, Polonais, Scandinaves, Néerlandais et Britanniques se rencontraient, non sans rixes et conflits, et parfois de violences, pouvant aller jusqu'à entraîner la mort. Le bouillant Dirck Schaep lui-même avait donné " un sufflet " à l'écuyer Du Meni[1], gendre de Monsieur de La Porte, qui dès lors lui en voulut à mort et le poursuivit de sa haine au point d'obliger Schaep à se cacher pour de bon. Joan se fait lui-même voler son argent à Chinon (il perd 50 florins, et Dirck encore 42 florins) et dépense 5 florins pour récupérer son dû. Il perd d'ailleurs assez souvent de l'argent tombé de sa poche, sommes qu'il enregistre avec précision.

Les comptes de Joan le jeune nous renseignent en particulier sur les détails et les prix de ses exercices. Ayant déjà fait quatre mois d'équitation dans le manège de Frobenius à Genève, il reprend maintenant ses exercices à Saumur. C'est en effet l'équitation qui y est au centre de ses soucis, et elle lui coûte assez cher. Il détaille l'entretien de ses chevaux, leur logement à l'écurie, d'abord dans l'auberge de La Corne [de Cerf], chez le sieur Prieur, et chez son hôte Gotiers, puis chez monsieur Chevrières, l'achat des fers, des éperons et de la têtière, voire les frais médicaux pour leur guérison, près de 25 florins, plus chers que ses propres dépenses médicales. Fin décembre, il a un maître de langue, un maître de luth et de guitare, chez qui il va pendant tout son séjour, et un maître de danse (deux fois un demimois). Le luth aussi est une source de dépenses continuelles. S'il va assez souvent à l'auberge, le plus gros de ses dépenses de loisirs concerne cependant les jeux de société : le jeu de paume, le jeu de maille et le jeu de piquet. Apparemment c'est surtout au jeu de paume qu'il perd régulièrement des sommes importantes au profit de ses compagnons néerlandais Schaep, Van der Duyn et autres, et il semble qu'il n'ait joué pour de l'argent qu'avec 
des compatriotes. D'ailleurs, le registre de l'académie mentionne au moins une occasion où le jeu de paume a dérapé en querelle sanglante entre un étudiant français et un gentilhomme anglais ${ }^{39}$.

L'apprentissage d'un français de qualité l'occupe également. Joan le jeune avait déjà appris l'espagnol, ce qui le gêne maintenant dans le perfectionnement du français, la solution étant, comme il l'écrit le 8 octobre à son père, de se loger dans un milieu exclusivement francophone. Il trouve aussi un maître de français et lorsque celui-ci le quitte pour la prestigieuse académie d'équitation d'Angers, il en trouve fin novembre un autre qui lui prend 30 florins. Les ambitions et le contrôle tatillon de son père, qui l'inonde de lettres d'avertissement, ont beaucoup travaillé le jeune Joan Huydecoper pendant son séjour à Saumur. Il est vrai que ce grand tour coûtait une petite fortune, 14000 florins en tout d'après le compte final de son père, soit plus que le prix d'une somptueuse demeure sur le canal le plus huppé d'Amsterdam. Le 28 décembre, accusant réception de trois lettres paternelles à la fois, il répond que le mécontentement sempiternel de son père l'a " réduit en desespoijr de vous contenter iamais ". Sur le reproche qu'il " fréquente la companie des vagabonts ", il énumère les personnes avec qui il est en contact à Saumur : tout d'abord le fils de M. Diodati de Genève, qui est le gouverneur de trois frères gentilshommes français, puis " des messieurs de cette ville " (donc l'élite saumuroise), enfin toute une série d'étudiants ou touristes hollandais qui y séjournent : Van Donselaer d'Amsterdam, Barck et son cousin d'Utrecht, Van der Vliet de La Haye, Van der Scheur (" fils de celui-là chez qui j'ai demeuré "), Driewegen et son frère, Rosiers de Zélande, Ravensway cousin de feu le général Van Diemen, Schrijver [mieux connu sous son nom latinisé de Scriverius] d'Amsterdam, Blaulaecken de Hoorn, " et Mond. frères d'Utrecht ".

\section{Pieter de la Court, le théologien manqué}

Contrairement à Huydecoper, l'autre voyageur qui a laissé des notes abondantes sur Saumur, Pieter de la Court, s'est bien intéressé à l'académie protestante, et cela d'autant qu'il avait encore des velléités dans le domaine des études théologiques ${ }^{40}$. En raison des frais considérables qu'un voyage de longue durée à l'étranger entraînait, le grand tour était en fait réservé aux jeunes bourgeois issus des couches régentes ou aux fils de négociants fortunés. Aucun de ces deux groupes n'était particulièrement intéressé par la profession de pasteur, mal rémunérée et au statut social assez moyen, en dépit des ambitions théologico-politiques de maint pasteur. Le nombre de ministres réformés néerlandais qui ont étudié à Saumur semble par

39. Arch. mun. de Saumur, Papier et regestre des affaires de l'académie royale, fo $164 \mathrm{v}^{\mathrm{o}}$ (au 12 juillet 1656).

40. Pour le grand tour comme marqueur de l'identité religieuse, voir VERHOEVEN, Gerrit, "Calvinist Pilgrimages and Popish Encounters : Religious Identity and Sacred Space on the Dutch Grand Tour (1598-1685) ", Journal of Social History, t. 43 (2010), p. 615-634. 
conséquent réduit. Il était régi par la disponibilité de bourses d'études, ou la possibilité de trouver un poste de précepteur de compagnie, ce qui permet de retrouver certains noms de jeunes étudiants en théologie dans le contexte d'un voyage de grande ampleur.

Il y a des exceptions. Tel Jacobus Revius (1586-1658), fils d'un bourgmestre de Deventer et futur pasteur dans sa ville natale, le centre culturel de la province d'Overijssel, où il contribua à la création de l'école illustre en 1630. Calviniste pur et dur, théologien contra-remontrant ${ }^{41}$ et anticartésien, mais aussi un des traducteurs de la Bible dite des États (Statenbijbel, 1637) et un poète de qualité, Revius est finalement nommé régent du collège théologique des États à Leyde. Dans ses notes autobiographiques il raconte comment en 1610, après ses études de théologie à Leyde et Franeker, "Galliae visendae avidus ", il prend le bateau pour Rouen, gagne Paris puis Orléans par la route, pour suivre la Loire jusqu'à Saumur. Outre le gouverneur Du Plessis-Mornay, il y fréquente les savants : "viri docti ac celebres in ea [Academia reformata] tunc florebant D. Cragius [Craig], D. Bodius Trochoregius [Boyd of Trochregd], Scoti, D. Boucherellus Minister, aliique non pauci ". Il fait les excursions habituelles à l'amphithéâtre de Doué, à Loudun, au collège jésuite de La Flèche et au Mans. Revenu à Saumur, il prend congé du gouverneur et poursuit son grand tour vers La Rochelle, Bordeaux et Montauban, pour rentrer par Cahors, Limoges, Poitiers et de nouveau Saumur, qui était décidément sa ville de référence. Deux ans après le début de son voyage il rentre chez lui ${ }^{42}$.

Vers la même époque, un autre jeune Néerlandais, Isaac Beeckman, passe lui aussi un temps d'études à Saumur. Son curriculum est caractéristique des jeunes ambitieux issus d'un milieu culturellement limité mais sachant saisir les occasions que la nouvelle société encore ouverte leur offre. Né à Middelbourg en Zélande, le 10 décembre 1588, fils aîné d'un maître cirier, Isaac débute en 1607 à Leyde dans la philosophie et les lettres, avec l'intention de continuer dans la théologie et se faire pasteur. Mais son véritable intérêt personnel était plus diversifié, tourné vers la philosophie naturelle et les sciences exactes et il s'est fait connaître plus tard par ses rapports avec Descartes, qui lui a d'ailleurs emprunté quelques-unes de ses idées maîtresses. Dès le début de ses études, Isaac tient un journal qu'il continue tout au long de sa vie. Nous savons ainsi qu'après ses débuts en théologie, il a passé son diplôme de maître cirier, pour se rendre au printemps de 1612 à Saumur où il achève ses études de théologie vers la fin de cette année. Pas question de grand tour pour cet artisan désargenté. Rentré chez lui, à défaut de pouvoir obtenir un poste de pasteur, il s'établit comme maître cirier et fontainier, tout en étudiant la médecine en autodidacte. Pendant l'été de 1618 il se rend de nouveau en France où il obtient le grade de licencié puis, le 6 septembre 1618, celui de docteur en médecine

41. Partisan de Gomar (n.d.l.r.).

42. Il détaille son itinéraire dans Revius, Jacobus, Daventria illustrata. Leyde, Petrus Leffen, 1651, p. 725-727; cf. DiBon, Paul, « Le voyage... », art. cit., p. 120. 
à l'université de Caen. Il poursuit alors sa carrière comme enseignant dans les fonctions de précepteur puis de recteur aux écoles latines d'Utrecht, Rotterdam et Dordrecht, où il meurt le 9 mai 1637 de la tuberculose ${ }^{43}$.

Dans la liste des thèses de théologie soutenues à Saumur, on ne trouve que deux noms néerlandais, à savoir Petrus Sylvius (1610-1653, fils du pasteur amstellodamois Johannes Sylvius), qui soutint sa thèse en 1636 et fut lui-même nommé pasteur à Muiden près d'Amsterdam en 1640, et Adrianus Hessels d'Utrecht, qui soutint en $1651^{44}$. Mais le Synode wallon donnait parfois une bourse d'études à des candidats prometteurs pour aller étudier à Saumur, tel Jacques Wattelier qui y fut envoyé en septembre 1614 pour six mois afin de perfectionner son français et poursuivre ses études en théologie ${ }^{45}$. Cependant, l'influence de Saumur sur le débat théologique en Hollande dépassait de loin la présence de ces quelques étudiants néerlandais ou wallons à l'Académie, même si ceux-ci servaient souvent de relais aux échanges d'idées. Tout au long du XvII ${ }^{\mathrm{e}}$ siècle, les positions modérées des théologiens saumurois dans la querelle de la grâce universelle, marquées par leur orientation fondamentalement humaniste, en particulier celles de John Cameron (1579-1625) et de ses disciples et successeurs Moïse Amyraut (1596-1664) avec son Bref traité de la prédestination (1634), l'exégète et philologue Louis (ou Ludovic) Cappel (1585-1658), Josué de La Place (vers 1596-1665) et Claude Pajon (1626-1685), ont une grande influence en Hollande ${ }^{46}$. Dès la fin des années 1640 le conflit y culmine dans une controverse autour des prises de position d'Alexander Morus (1616-1670), qui, originaire d'Écosse mais né à Castres et devenu professeur de théologie à Genève, cherche alors un poste de professeur dans la République. En tant que partisan d'Amyraut, sa candidature est rejetée par

43. VAN BERKEL, Klaas, Isaac Beeckman (1588-1637) en de mechanisering van het wereldbeeld/Isaac Beeckman and the Mechanization of the World Picture, Amsterdam, Rodopi, 1983.

44. Sintagma thesium theologicarum in Academia Salmuriensium variis temporibus disputatarum, ed. secunda, Saumur, apud J. Lesnerium, 1665, t. I, p. 117-143; DESGRAVES, Louis, "Les thèses soutenues à Académie protestante de Saumur au XVII ${ }^{\mathrm{e}}$ siècle ", Bulletin de la Société de l'Histoire du Protestantisme Français, t. 125 (1979), p. 76-97, ici p. 86, nº 37, et p. $89, \mathrm{n}^{\circ} 58$. Hessels ne figure pas dans le répertoire des pasteurs réformés établi par Fred van Lieburg; il peut s'agir d'un fils ou petit-fils d'Adrianus Hesselius qui fut doyen réformé du chapitre sécularisé de Saint-Jean d'Utrecht depuis 1619 et mourut avant 1633.

45. Dibon, Paul, "Le voyage... ", art. cit., p. 140, note 93. Voir plus amplement le Livre synodal contenant les articles résolus dans les Synodes des Églises Wallonnes des Pays-Bas. Tome I : 1563-1685, La Haye, Martinus Nijhoff, 1896.

46. LAPLANCHE, François, Orthodoxie et prédication. L'œuvre d'Amyraut et la querelle de la grâce universelle, Paris, PUF, 1965; du même, "Tradition et modernité au XVII ${ }^{\mathrm{e}}$ siècle : l'exégèse biblique des protestants français ", Annales ESC, t. 40 (1985), p. 463-488; du même, L'écriture, le sacré et l'histoire : érudits et politiques protestants devant la Bible en France au XVII siècle, Amsterdam \& Maarssen, APA, 1986; KRETZER, Hartmut, Calvinismus und französische Monarchie im 17. Jahrhundert. Die politische Lehre der Akademien Sedan und Saumur, Berlin, Duncker \& Humblot, 1975; van STAM, Frans Pieter, The Controversy over the Theology of Saumur, 1635-1650: Disrupting Debates among the Huguenots in Complicated Circumstances, Amsterdam, Holland University Press, 1988. 
plusieurs académies néerlandaises avant être finalement acceptée en 1650 par la nouvelle École illustre de Middelbourg ${ }^{47}$.

La voie moyenne prônée par ce qui est resté connu comme l'École (théologique) de Saumur - ce « calminianisme ", mélange de calvinisme et d'arminianisme qui en est issu, bientôt associé au cartésianisme-, demeurait jusqu'au XVIII ${ }^{\mathrm{e}}$ siècle l'alternative critique et tolérante au calvinisme néerlandais sévère de la double prédestination formulée au Synode de Dordrecht (1618-1619), au point qu'il inspira encore au milieu du XVIII ${ }^{\mathrm{e}}$ siècle le «Dessein de tolérance " formulé au sein de cette Église pour réconcilier le calvinisme orthodoxe avec la position arminienne des remontrants ${ }^{48}$. Cette voie moyenne, loin de toute intransigeance, est probablement ce qui a séduit à Saumur le jeune Pieter de la Court. Son journal de voyage et ses carnets de notes sont d'une force d'observation inhabituelle et d'un caractère personnel prononcé qui les détachent des documents parsemés de stéréotypes et d'observations anodines courants parmi la gent pérégrine ${ }^{49}$.

Pieter de la Court le jeune était né à Leyde en 1618, second fils de Pieter de la Court l'aîné (1593-1657), un immigré d'Ypres, prospère fabricant et négociant de drap dans sa ville d'adoption qui fut probablement un vrai réfugié pour la foi, réformé zélé et ancien de l'Église wallonne de Leyde qui était alors la deuxième ville du pays, après Amsterdam ${ }^{50}$. Inscrit à l'université de Leyde en 1631 comme élève des classes supérieures du collège, Pieter y accomplit ensuite ses études universitaires avant d'entreprendre un grand tour. Du 25 septembre 1641 au 30 octobre 1643, celui-ci le conduit d'abord en Angleterre, puis le 4 avril 1642 à Dieppe. Il passe ensuite par Paris et Orléans pour se rendre à Saumur. Il y reste deux mois, du 28 avril au 30 juin 1642. De là il voyage jusqu'à Lyon et Marseille, mais le voyage prévu en Italie est finalement annulé en faveur d'un séjour de neuf mois à Genève puis d'un autre mois à Bâle et de quelques semaines à Francfort et à Cologne. Les universités et académies réformées dominent clairement

47. Sur lui : Nieuw Nederlandsch Biografisch Woordenboek, t. X (1937), col. 652-653; EuIAS, J.E., De vroedschap van Amsterdam, t. II, p. 822-825.

48. GraAfland, Cornelis, "Examen van het Ontwerp van Tolerantie ", Theologia Reformata, t. 15 (1972), p. 20-36.

49. Les documents originaux se trouvent dans Regionaal Archief Leiden, Familiearchief De la Court, $\mathrm{n}^{\circ} 14$ (Journal et notes de voyage, 1641-1643). Le journal a été publié par DRIESSEN, Felix, De reizen der De la Courts, 1641-1700-1710, s.l., s.d. [Leyde, 1928], p. 1-30; les notes de voyage, ainsi qu'une évaluation du journal, par FRIJHOFF, Willem, "Pieter de la Courts reisjournaal (1641-1643) als ego-document " et " De reisnotities (1641-1643) van Pieter de la Court. Uit het manuscript bezorgd en van commentaar voorzien ", in Blom, Hans W. et Wildenberg, Ivo W. (dir.), Pieter de la Court in zijn tijd. Aspecten van een veelzijdig publicist, Amsterdam \& Maarssen, APA-Holland University Press, 1986, p. 11-34 et 35-64 (p. 41-46 concernent des notes prises à Saumur). Voir DiBon, Paul, "Le voyage... ", art. cit., p. 130, et van Westrienen, Frank, De Groote Tour, passim.

50. Notice biographique dans Nieuw Nederlandsch Biografisch Woordenboek, t. VII, Leyde, Sijthoff, 1927, col. 335-337; WILDENBERG, Ivo W., Johan en Pieter de la Court (1662-1660 en 1618-1685). Bibliografie en receptiegeschiedenis, Amsterdam \& Maarssen, APA-Holland University Press, 1986; WestSTEIn, Arthur, Commercial Republicanism in the Dutch Golden Age : The Political Thought of Johan \& Pieter de la Court, Leyde et Boston, Brill, 2012. 
ce voyage et expriment l'intérêt que Pieter de la Court prend à la théologie. D'ailleurs, dès son retour, le 10 novembre 1643 , il reprend ses études de théologie à Leyde, sans toutefois persister dans cette voie. Établi dans l'industrie drapière de son père, puis à son propre compte avec son frère cadet Johan (1622-1660), il occupe son temps libre à écrire les traités économiques et politiques de facture libérale et républicaine, clairement anti-orangiste, qui ont établi sa renommée comme un des plus importants penseurs de l'économie politique et des régimes politiques du XVII ${ }^{\mathrm{e}}$ siècle. En 1670 il se rend à Orléans pour prendre un grade en droit, puis s'établit comme avocat à Amsterdam, où il est également un des dix-neuf directeurs de la compagnie des Indes Occidentales. Lors de l'invasion française de 1672 il doit provisoirement se réfugier à Anvers, par crainte d'une revanche des Orangistes. Il meurt à Amsterdam le 28 avril 1685.

Le journal et les notes de Pieter de la Court nous renseignent abondamment sur les étudiants et touristes néerlandais qui l'accompagnent ou qu'il rencontre en route, à commencer par ses deux compagnons fixes à Saumur, l'étudiant en théologie Abraham de Matter (1617-1669), natif de Leyde et qui y finira ministre, et Jan de Mey (1617-1678), natif de Middelbourg, un théologien accompli. Ce dernier, qui avait déjà été ministre dans un petit village de Zélande, l'accompagnera pendant tout son voyage à travers la France, prendra un grade en médecine à Valence et le persuadera à Marseille d'échanger la catholique Italie pour la protestante Genève. Jan de Mey, piétiste plus philosophe que théologien, était un neveu de l'ancien élève de Saumur Isaac Beeckman. En 1650, il est nommé pasteur dans sa ville natale de Middelbourg, puis lecteur en philosophie et professeur de théologie à l'École illustre de cette ville, où il se signalera par une forte réflexion spéculative sur la philosophie naturelle ${ }^{51}$.

Les textes montrent aussi que le voyage a un double but pour Pieter de la Court : d'abord un objectif étudiant, puisque les villes universitaires sont son point d'ancrage, mais tout autant un but professionnel, car le texte abonde en observations sur l'industrie drapière. Autant en Angleterre qu'en France il regarde tout ce qu'il rencontre avec l'œil professionnel du fabricant de drap, détaillant toujours la qualité des étoffes et des produits, les habits, leurs mesures et leurs couleurs, les procédés de fabrication, agissant parfois quasiment comme un espion pour son père. Ayant regardé Paris pendant une semaine en vrai touriste, il se rend à Tours où il s'embarque avec son compagnon de voyage, Jan de Mey. Il s'assied à côté de deux cordeliers avec qui il aurait aimé discuter s'il n'y avait eu un grand nombre de femmes dans la barque; les moines qu'il rencontre alors sont à son avis "des gens sympathiques mais sans aucune culture ". Arrivé le soir à Saumur, il se loge pour la nuit à La Corne de Cerf, repaire habituel des Néerlandais. En fait, il aurait voulu être logé chez un des professeurs,

51. ZUIDERVAART, Huib J., « Het natuurbeeld van Johannes de Mey (1617-1678), hoogleraar filosofie aan de illustere school te Middelburg " : [http://www.huygens.knaw.nl/wp-content/ bestanden/pdf_Zuidervaart_2001_De_Mey.pdf]. 
comme le faisaient d'autres étudiants, mais il ne restait de place ni chez Louis Cappel ni chez Josué de La Place. Dès le lendemain, sur recommandation du médecin Jean Benoist, professeur de langue grecque, il trouve, avec son compagnon, un gite chez l'apothicaire Haupilet pour 25 florins par mois. Benoist et Cappel lui donneront d'ailleurs des lettres de recommandation lors de son départ.

Pendant son séjour à Saumur, son journal s'étoffe d'observations religieuses, beaucoup plus qu'à Genève, même s'il faut tenir compte du non-dit et des évidences, telles que la visite au temple dont il ne parle pas. Une des raisons en est certainement la situation de contact permanent entre catholiques et protestants dans la ville, et le dialogue religieux qui en résultait. Dans son pays d'origine, ce dialogue était occulté par le statut de minorité, voire l'interdit cultuel, dont souffraient les catholiques, mais à Saumur il y est constamment confronté et il donne libre cours à ses réflexions. Ainsi, il s'étonne de voir des moines qui mendient dans la rue, de préférence au cours de l'après-midi, de la multiplication des messes basses simultanées dans les églises, du nombre prodigieux d'autels - vingt-cinq au moins dans l'église de l'abbaye de Saint-Florent, mais aussi de l'ouverture des boulangeries, halles et auberges le dimanche et de l'afflux des paysans dans la ville pour vendre leurs produits les jours de fête, l'artisanat étant interdit le dimanche mais pas le commerce. L'arrêt subit des Saumurois catholiques dans la rue, au son de la cloche à midi et à 7 heures du soir, pour demander pardon de leurs péchés, le surprend autant que l'usage abondant de clystères qu'il détecte chez les Angevins. Lors de l'orage mémorable du 18 juin 1642, les prêtres catholiques font, à son étonnement, sonner les cloches pour chasser le tonnerre. Les réformés de Saumur provoquent aussi quelques remarques étonnées car, curieusement, ils n'observent aucun des jours fériés, même pas Noël ou la Pentecôte, et ils ne choisissent pas de péricope ${ }^{52}$ du jour, deux omissions qui sont sans doute à imputer au désir de se démarquer des catholiques. Du côté catholique, il note le refus de porter des vêtements particuliers le jour du Seigneur.

Dès avant la visite de Saumur, lors de son parcours en Normandie, il s'était montré avide de contacts avec les rituels catholiques, qu'il rejetait bien sûr mais n'arrêtait pas de décrire à chaque occasion, fasciné qu'il était par une religiosité dont il n'arrivait pas à comprendre la rationalité. Outre les excursions obligées à l'amphithéâtre de Doué qu'il décrit en touriste sans montrer beaucoup de surprise, à La Flèche qui ne lui provoque aucun commentaire, même pas à propos des jésuites, ou à la grotte du poète Théophile de Viau qu'il vénère pour son imitation de la nature, il se rend, en revanche, avec une curiosité à peine retenue, à plusieurs reprises à NotreDame-des-Ardilliers dont il détaille avec précision le pèlerinage, les ex-voto, et même les coutumes populaires para-religieuses qui s'exercent hors du sanctuaire. Il refuse d'enlever son chapeau en présence de l'objet du culte, la statue miraculeuse, refus qui commence à devenir dangereux au moment

52. Passage des Livres saints lus à l'Évangile (ndlr). 
où la situation se détériore pour les réformés, mais il n'ose pas aller jusqu'à demander au religieux qui en est le gardien de pouvoir voir de près la statue dévoilée, en dehors du culte, donc en tant que touriste curieux, comme le fera son compagnon De Matter, plus courageux à cet égard.

Pendant le week-end de la Pentecôte, il se rend à plusieurs reprises aux Ardilliers et il détaille les dévotions populaires qu'il y observe autour de la statue et le toucher rituel des manteaux de la Vierge. D'autres fêtes communautaires avec leurs cérémonies religieuses et leurs cortèges dans la ville sont encore décrites avec précision, telle la fête des boulangers, le 17 mai, celle des tailleurs d'habits le dimanche de la Trinité, soit le 15 juin en 1642, ou encore les feux de la Saint-Jean le 24 juin. Le jeudi après la Trinité, le 19 juin, la procession publique de la Fête-Dieu est pour lui une vraie découverte du caractère éminemment confessionnel de la pratique de la foi catholique. Il la décrit quasiment en anthropologue, exactement comme l'enterrement d'un enfant auquel il assiste le lendemain, puis, quelques jours plus tard, celui d'un homme catholique de qualité. Il note d'ailleurs que les rapports entre les religions se détériorent, qu'on injurie les protestants en les invectivant comme " parpaillots ", et qu'il arrive que, sur la voie publique, les catholiques tirent les réformés par leur manteau pour les forcer à adorer un crucifix ou à se rendre à la messe, ce qui venait d'arriver au théologien Louis Cappel dont apparemment un fils s'était converti récemment au catholicisme. Lors de la Fête-Dieu, les réformés de Saumur avaient même été obligés d'accrocher des tapis à leurs maisons pour le passage de la procession du Saint-Sacrement.

Bien évidemment il veut en avoir le cœur net et discute avec les professeurs Louis Cappel et Moïse Amyraut de ses expériences de la religion catholique et de l'attitude qu'il doit adopter à cet égard. Ainsi, nous le voyons au petit matin du 2 juin, à cinq heures, en promenade savante avec Cappel aux environs de Saumur où il scrute les antiquités locales; il y discute aussi avec lui de la description du temple de Salomon par le jésuite espagnol Juan Bautista Villalpando et du commentaire écrit par Cappel sur cette reconstruction. D'autres sujets de discussion avec Cappel pendant leurs promenades sont la publication volumineuse de " vieux monuments " parcheminés par les jésuites romains, - peut-être un renvoi aux 12 volumes des Annales ecclesiastici du cardinal oratorien César Baronius (1538-1607), publiés à Rome entre 1588 et 1607 -, l'édition de la Septante par François du Jon ou Junius (1545-1602), professeur à Leyde, et les puits dans lesquels les vieux Hébreux tenaient captifs leurs esclaves, d'après Cappel qui invoque Zacharie 9:11. Amyraut de son côté lui conseille de lire la Theologia scholastica (Mayence, 1612) du jésuite brabançon Martinus Becanus (1563-1624) qui, à son avis, décrit le plus exactement la théologie catholique ${ }^{53}$. Il fait savoir à de La Court qu'à son avis le Christ avait institué la precatio dominica uniquement à l'usage de

53. Becanus, Martinus, Theologia scholastica, Mayence, 1612, ouvrage qui connut des dizaines de réimpressions. Becanus (1563-1624) était né à Hilvarenbeek au Brabant, entra 
ses disciples. Enfin, Pieter note aussi en quelques mots la position des Saumurois dans le grand conflit théologique qui sépare les factions des Églises réformées : "Jos. La Place en Mons. Amyrault negant peccatum Adami nobis imputari ut et justitiam Christ[i] passivam ", en ajoutant un peu plus tard «Idem quoque Cappel ${ }^{54}$ ".

La conclusion de ce tour d'horizon se doit de rester simple et ouverte. Situé au milieu d'un itinéraire devenu classique et offrant aux jeunes privilégiés de la société hollandaise toutes les conditions pour un séjour aussi utile qu'agréable, la ville de Saumur a joué pour eux un rôle beaucoup plus important dans la construction de réseaux de sociabilité et d'identification religieuse que dans l'étude de la théologie où elle n'a eu qu'un rôle de relais. Initialement l'académie s'est profilée pour eux comme une étape utile sur la route du savoir, mais assez rapidement le développement des universités et académies néerlandaises a pris le relais, d'autant que les querelles théologiques ont ébranlé le rôle de Saumur comme roc réformé. Il en restait une ville où il faisait bon vivre et où la jeunesse dorée internationale pouvait se rencontrer sans trop de contrôle par des parents inquiets ou tatillons ou par les autorités politiques et ecclésiastiques de leurs pays d'origine.

Figure 1 - Lambert Doomer (Amsterdam, 1624-1700), La ville et le château de Saumur, vus du Nord pendant son voyage en France, 1646; dessin achevé vers 1670 (The J. Paul Getty Museum, Los Angeles)

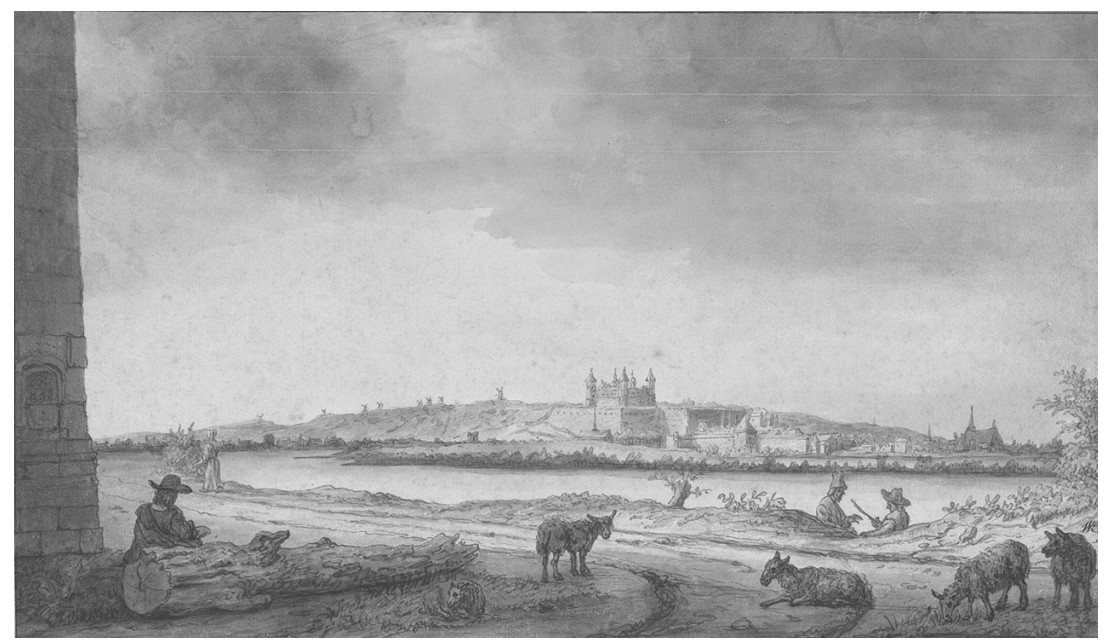

dans la Compagnie de Jésus, devint professeur de théologie à Würzburg, Mayence et Vienne, et finit comme confesseur de l'empereur Ferdinand II.

54. Notes de voyage, fo 51 vo (FriJHOFF, Willem, " De reisnotities (1641-1643)... ", art. cit., p. 45). 
Figure 2 - Exemple d'un itinéraire du Grand Tour passant par Saumur : le voyage d'études de Johan Kaldenbach, fils d'un bourgmestre de Zutphen (Gueldre), (1599-) 1604-1609 (Carte dessinée par l'auteur)

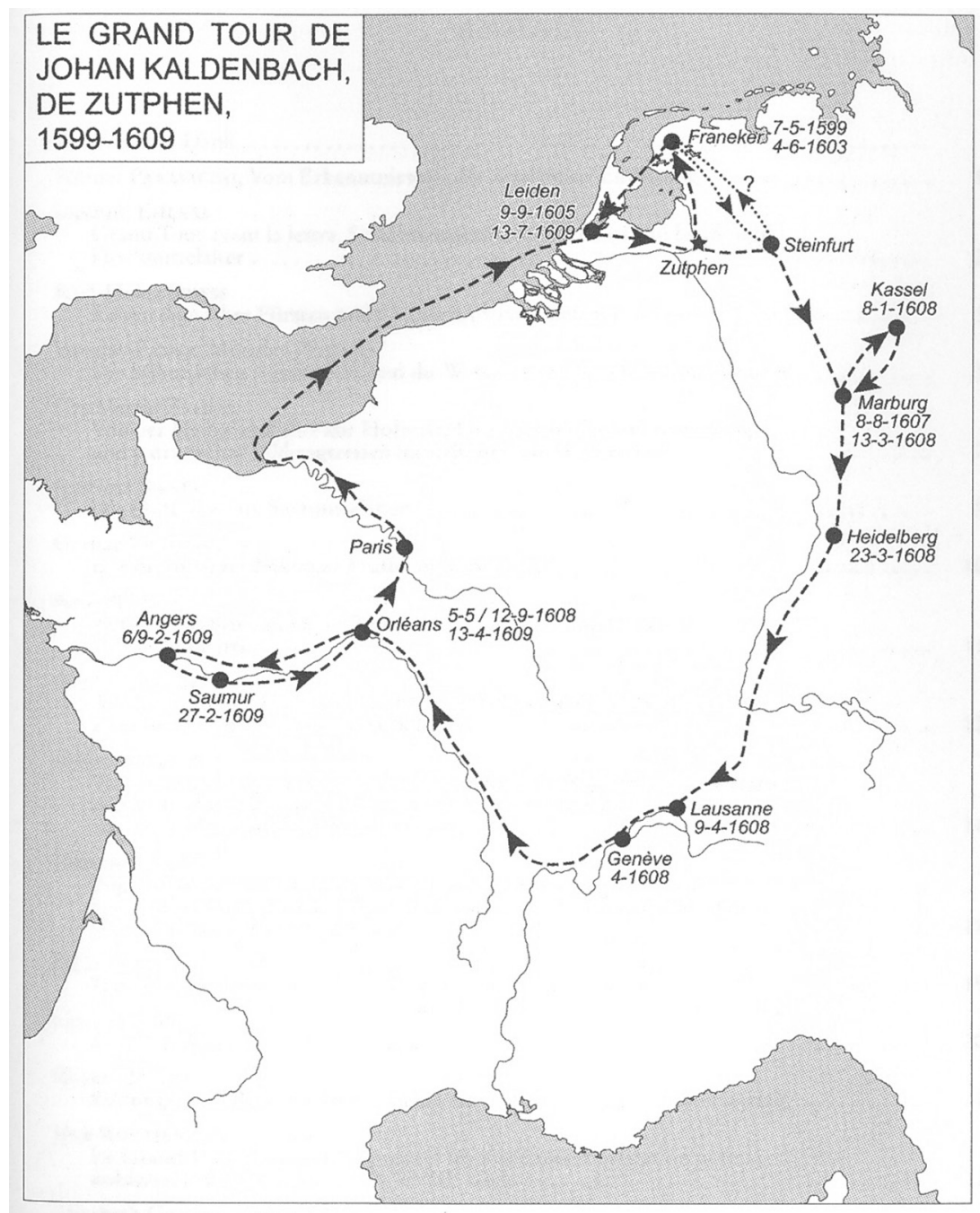



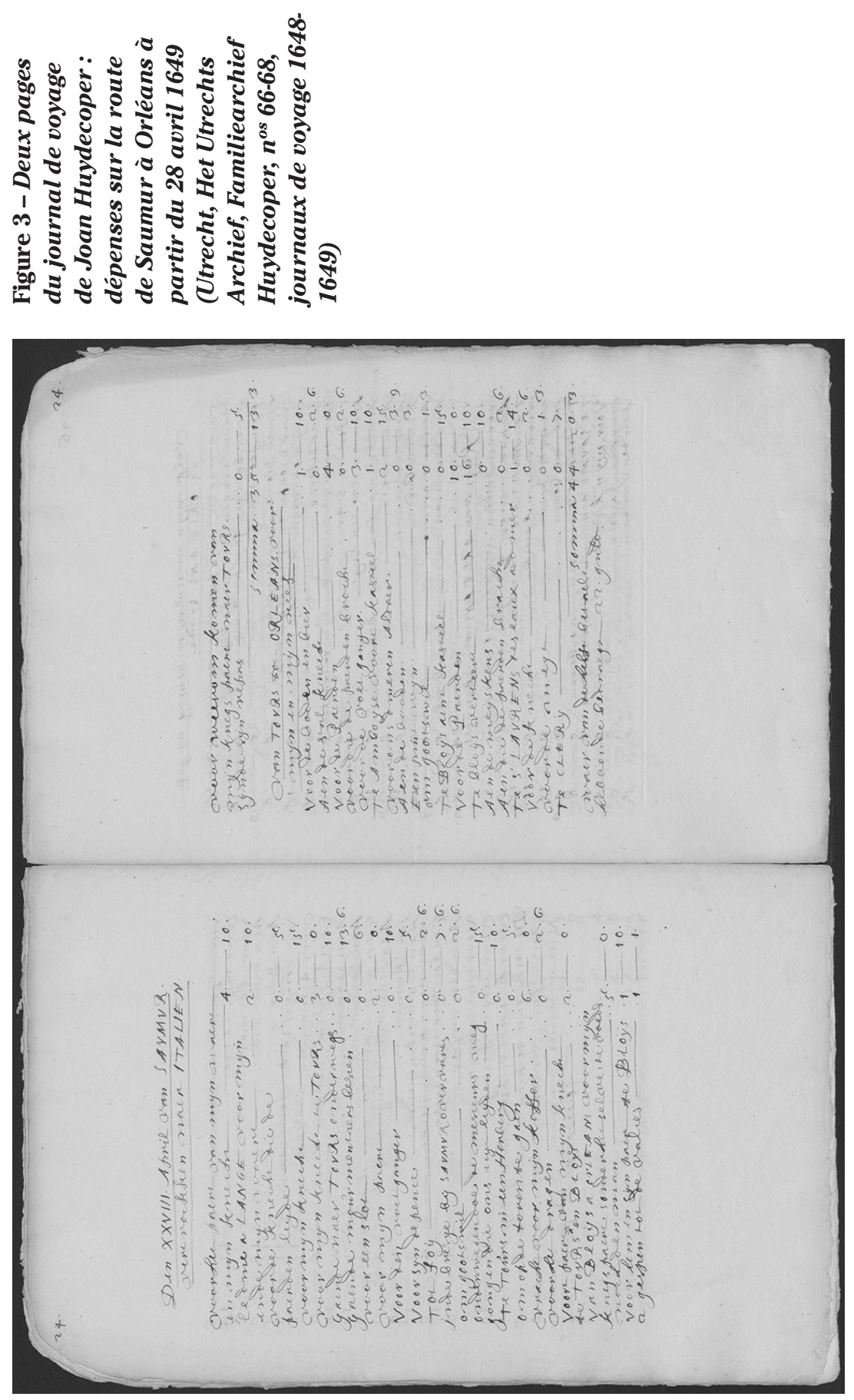
Figure 4 - Pieter de la Court en 1667, peinture par Abraham van den Tempel (1622-1672) (Rijksmuseum Amsterdam, SK-A-2243).

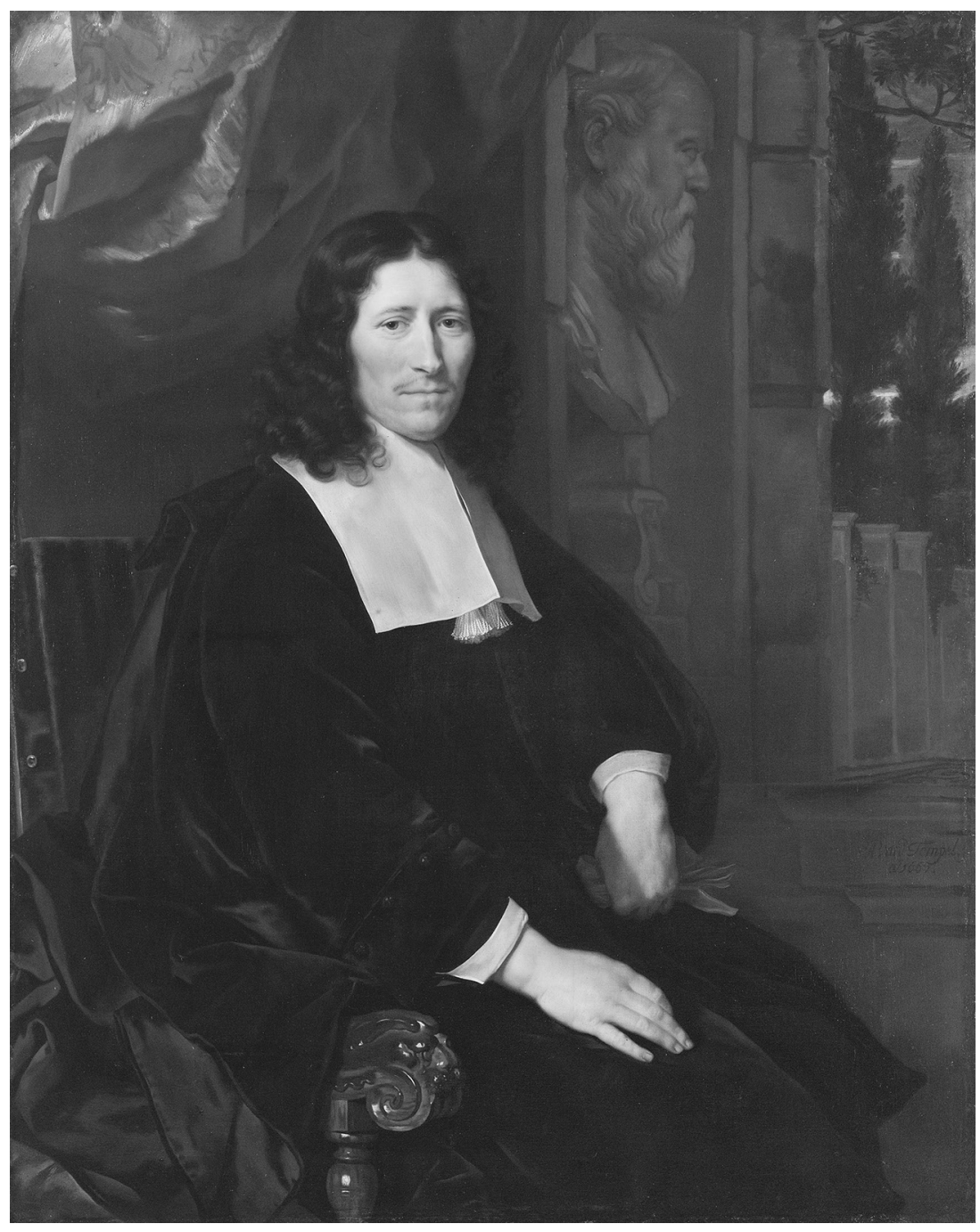




\section{RÉSUMÉ}

Au XVII ${ }^{\mathrm{e}}$ siècle, Saumur était une étape incontournable du grand tour des jeunes Néerlandais, tout d'abord pour une visite ou un séjour d'études à l'Académie protestante qui y a fonctionné jusqu'à la Révocation, mais tout autant en raison du climat agréable, des maîtres particuliers qui y offraient leurs services, du vin d'Anjou et de la pureté reconnue de la langue française que l'on pouvait y apprendre, sans oublier l'académie des Pères de l'Oratoire aux Ardilliers. Saumur était pour les Néerlandais une ville multifonctionelle hautement appréciée qui attirait étudiants, futurs pasteurs et prêtres, savants, marchands, touristes fortunés ou simples passants. À défaut d'un livre matricule (= liste des étrangers ayant fréquenté Saumur, explication pour la traduction, AA), on trouvera des témoignages détaillés et éloquents dans les sources privées de plusieurs grands noms du Siècle d'Or hollandais. Cet article dresse un bilan de l'importance de Saumur pour les Néerlandais avant d'analyser en détail le journal de voyage de Pieter de la Court, fils d'un drapier de Leyde, théologien manqué et important auteur politique républicain, qui y séjourna en 1642, ainsi que la correspondance de Joan Huydecoper avec son père, puissant bourgmestre d'Amsterdam, et le journal comptable qu'il a tenu pendant les neuf mois de son séjour à Saumur en 1648-1649, sous la Fronde.

\section{ABSTRACT}

During the $17^{\text {th }}$ century, Saumur was a compulsory stage on the Grand Tour of the Dutch, not only because of the local Protestant Academy active until the Revocation of the Edict of Nantes, but also for its pleasant climate, its private teachers supplying a wide range of services, the Anjou wine, and the well-known purity of the French language used in that region - not to forget the Catholic academy of the Oratorian fathers at Notre-Dame des Ardilliers. For the Dutch, Saumur was a multifunctional town, much appreciated by students on their Grand or Petit Tour, by future ministers, priests, scholars and merchants, and by wealthy tourists and foreign passers-by. The absence of matriculation registers is partly compensated by a range of private documents stemming for some of the greatest names of the Dutch Republic. This article takes stock of Saumur's interest for the Dutch. It draws up a synthesis of the available data before analysing in detail two private sources: a travel journal that young Pieter de la Court, son of a cloth merchant of Leiden, a failed theology student and an influential political and republican author, held during his grand tour in 1642, and the correspondence of Joan Huydecoper with his father, a powerful burgomaster of Amsterdam, including his account book during his nine months stay at Saumur in 1648-1649, at the time of the Fronde. 